\title{
Metallicity effect on stellar granulation detected from oscillating red giants in open clusters
}

\author{
E. Corsaro ${ }^{1,2,3,4}$, S. Mathur ${ }^{5}$, R. A. García ${ }^{4}$, P. Gaulme ${ }^{6,7,8}$, M. Pinsonneault ${ }^{9}$, K. Stassun ${ }^{10}$, D. Stello ${ }^{11,12,13}$, \\ J. Tayar ${ }^{9}$, R. Trampedach ${ }^{5,13}$, C. Jiang ${ }^{14}$, C. Nitschelm ${ }^{15}$, and D. Salabert ${ }^{4}$ \\ 1 INAF-Osservatorio Astrofisico di Catania, via S. Sofia 78, 95123 Catania, Italy \\ e-mail: enrico.corsaro@oact.inaf.it \\ 2 Instituto de Astrofísica de Canarias, 38205 Tenerife, Spain \\ 3 Universidad de La Laguna, Departamento de Astrofísica, 38206 La Laguna, Tenerife, Spain \\ ${ }^{4}$ Laboratoire AIM, CEA/DRF - CNRS - Univ. Paris Diderot - IRFU/SAp, Centre de Saclay, 91191 Gif-sur-Yvette Cedex, France \\ 5 Space Science Institute, 4750 Walnut street Suite\#205, Boulder CO 80301, USA \\ ${ }^{6}$ Department of Astronomy, New Mexico State University, PO Box 30001, MSC 4500, Las Cruces, NM 88003-8001, USA \\ 7 Apache Point Observatory, 2001 Apache Point Road, PO Box 59, Sunspot, NM 88349, USA \\ 8 Physics Department, New Mexico Institute of Mining and Technology, 801 Leroy Place, Socorro, NM 87801, USA \\ 9 Department of Astronomy, Ohio State University, 140 W 18th Ave, OH 43210, USA \\ 10 Department of Physics and Astronomy, Vanderbilt University, 1807 Station B, Nashville, Tennessee 37235, USA \\ 11 School of Physics, University of New South Wales, NSW 2052, Australia \\ 12 Sydney Institute for Astronomy (SIfA), School of Physics, University of Sydney, NSW 2006, Australia \\ 13 Stellar Astrophysics Centre, Department of Physics and Astronomy, Aarhus University, Ny Munkegade 120, 8000 Aarhus C, \\ Denmark \\ 14 Instituto de Astrofísica e Ciências do Espaço, Universidade do Porto, CAUP, Rua das Estrelas, 4150-762 Porto, Portugal \\ 15 Unidad de Astronomía, Universidad de Antofagasta, Avenida Angamos 601, 1270300 Antofagasta, Chile
}

Received 3 May 2017 / Accepted 19 July 2017

\begin{abstract}
Context. The effect of metallicity on the granulation activity in stars, and hence on the convective motions in general, is still poorly understood. Available spectroscopic parameters from the updated APOGEE-Kepler catalog, coupled with high-precision photometric observations from NASA's Kepler mission spanning more than four years of observation, make oscillating red giant stars in open clusters crucial testbeds.

Aims. We aim to determine the role of metallicity on the stellar granulation activity by discriminating its effect from that of different stellar properties such as surface gravity, mass, and temperature. We analyze 60 known red giant stars belonging to the open clusters NGC 6791, NGC 6819, and NGC 6811, spanning a metallicity range from $[\mathrm{Fe} / \mathrm{H}] \simeq-0.09$ to 0.32 . The parameters describing the granulation activity of these stars and their frequency of maximum oscillation power, $v_{\max }$, are studied while taking into account different masses, metallicities, and stellar evolutionary stages. We derive new scaling relations for the granulation activity, re-calibrate existing ones, and identify the best scaling relations from the available set of observations.

Methods. We adopted the Bayesian code DIAMONDS for the analysis of the background signal in the Fourier spectra of the stars. We performed a Bayesian parameter estimation and model comparison to test the different model hypotheses proposed in this work and in the literature.

Results. Metallicity causes a statistically significant change in the amplitude of the granulation activity, with a dependency stronger than that induced by both stellar mass and surface gravity. We also find that the metallicity has a significant impact on the corresponding time scales of the phenomenon. The effect of metallicity on the time scale is stronger than that of mass.

Conclusions. A higher metallicity increases the amplitude of granulation and meso-granulation signals and slows down their characteristic time scales toward longer periods. The trend in amplitude is in qualitative agreement with predictions from existing 3D hydrodynamical simulations of stellar atmospheres from main sequence to red giant stars. We confirm that the granulation activity is not sensitive to changes in the stellar core and that it only depends on the atmospheric parameters of stars.
\end{abstract}

Key words. stars: oscillations - methods: numerical - methods: statistical - stars: late-type - open clusters and associations: general - stars: fundamental parameters

\section{Introduction}

Granulation is a type of stellar variability and it is a surface manifestation of stellar envelope convection. Here hot gas in the granules rises from the interior to the photosphere where the thermal energy of the granules is lost to the radiation field, reaching velocities comparable to the local sound speed. The cooled, denser plasma is thus pushed to the edges of the granules and sinks back into the star in the darker inter-granular lanes. According to this interpretation, a characteristic time scale for the phenomenon is to first approximation given as $\propto \sqrt{T_{\text {eff }}} / g$ (Brown et al. 1991; Kjeldsen \& Bedding 2011), where $g$ is the surface gravity of the star. For solar-like oscillating stars, acoustic oscillations also originate from the turbulent motions caused by convection, although granulation remains the dominant component in terms of energy that is visible at the stellar surface because the intensity 
fluctuation related to granulation can be up to about three times that related to the acoustic oscillation signal (Kallinger et al. 2014, hereafter K14).

The study of stellar granulation was born through its observation on the Sun (Herschel 1801). The first analysis using the Fourier approach to measure the granulation time scale and amplitude was done by Harvey (1985), and subsequently improved by for example, Aigrain et al. (2004). Since then, granulation activity has been observed in a large variety and number of lowand intermediate-mass stars with convective envelopes (e.g. Kallinger \& Matthews 2010; Mathur et al. 2011; Hekker et al. 2012; Karoff et al. 2013; K14). It has also been used to obtain accurate model-independent measurements of stellar surface gravity (Bastien et al. 2013, 2016; Kallinger et al. 2016). These studies have been made possible thanks to the advent of high-precision photometry from space missions such as CoRoT (Baglin et al. 2006) and NASA Kepler (Borucki et al. 2010), the latter having been used to observe more than 197000 stars (Mathur et al. 2017). These space missions provided both sampling rates rapid enough for resolving the typical time scales of granulation, and observing lengths that allowed for characterization of the granulation properties to a high degree of precision and accuracy. In particular, the first ensemble study was done for red giants (RGs) by Mathur et al. (2011) using Kepler data spanning more than one year of nearly-continuous observations. The authors showed that the granulation power and time scale are strongly correlated with the frequency of maximum oscillation power, $v_{\max }$, the latter scaling with the acoustic cut-off frequency of the star (Brown et al. 1991). Later K14 provided a thorough calibration of these dependencies by extending the sample to main sequence stars and using Kepler observations covering more than three years.

Studying the connection between the granulation signal and fundamental stellar properties such as surface gravity, mass, temperature, and chemical composition is essential to better understand convection in stars. A better understanding of stellar granulation can yield more detailed descriptions of turbulent motions in stellar atmospheres, and therefore improve stellar structure and evolution models. More realistic stellar models improve our capability to retrieve accurate stellar properties, and provide high-quality evolution sequences for ensemble analysis of, for example, the Galactic formation and evolution, especially in view of the ESA Gaia mission (Perryman et al. 2001). Efforts in this direction have been made from a theoretical point of view by using 3D hydrodynamical models of stellar atmospheres (e.g., Trampedach et al. 1998, 2013, 2014; Ludwig 2006; Mathur et al. 2011; Samadi et al. 2013a,b), although only a few studies (e.g., Collet et al. 2007; Magic et al. 2015b,a) have dealt with metallicity effects on such 3D simulations of convective atmospheres. As shown by Collet et al. (2007) for RGs (see also the work by Tanner et al. 2013, on gray atmospheres of main sequence stars), stellar metallicity appears to play an important role in determining the scale of granulation, yielding larger granules as metallicity increases, hence a higher amplitude of the associated granulation signal (Ludwig 2006). This result has been further confirmed for evolved stars by Ludwig \& Steffen (2016). However, any observational evidence of the metallicity effect on granulation has neither been found nor discussed in the literature until now.

Stellar clusters offer a possibility to exploit the accurate knowledge of the common physical properties shared by their members. The open clusters NGC 6791, NGC 6819, and NGC 6811 have been monitored by the Kepler mission for more than four years, thus providing us with the best photometric observations currently available for the rich populations of RGs hosted by each of these clusters (Stello et al. 2011). Fundamental parameters such as temperature, mass, metallicity, and age, are determined for cluster stars with high reliability (e.g., Bragaglia et al. 2001; Basu et al. 2011; Hekker et al. 2011; Stello et al. 2011; Brogaard et al. 2011, 2012; Miglio et al. 2012), and the evolutionary stage of many cluster RGs is also well known from existing asteroseismic analyses (Corsaro et al. 2012; Mosser et al. 2014; Vrard et al. 2016; Corsaro et al. 2017).

In this work we have exploited the full Kepler nominal mission photometric data for the open clusters NGC 6791, NGC 6819 and NGC 6811, and the wealth of spectroscopic observations available from APOKASC (Pinsonneault et al. 2014), to properly disentangle the effect of metallicity from that of other fundamental stellar properties by performing a thorough Bayesian approach that takes into account uncertainties on all the observables. In this way we will assess the behavior of granulation activity in RGs in light of existing theoretical predictions.

\section{Observations and data}

\subsection{Sample selection and photometry}

The sample of RGs of the open clusters NGC 6791 and NGC 6819 is derived from the original set of 111 stars analyzed by Corsaro et al. (2012). We included those stars with a clear evolutionary stage determination, as discriminated using mixed mode oscillations (Bedding et al. 2011) by Corsaro et al. (2012), Mosser et al. (2014), Corsaro et al. (2017; see also Corsaro et al., in prep.). We find in total 30 RGs for NGC 6791 and 24 for NGC 6819. For NGC 6811, we considered the four stars with a known evolutionary stage from Corsaro et al. (2012) and we added two more, KIC 9776739 and KIC 9716090, analyzed by Molenda-Żakowicz et al. (2014) and by Corsaro et al. (in prep.) and both classified as core-He-burning RGs (red clump stars, hereafter $\mathrm{RC}$ ), thus reaching a total of six targets for this cluster. The final sample therefore accounts for $60 \mathrm{RGs}$, with $38 \mathrm{RC}$ stars and 22 shell-H-burning RGs (red giant branch, hereafter RGB).

The photometric observations for the selected sample of stars were conducted by NASA's Kepler telescope in the observing quarters (Q) from Q0 till Q17, for a total of $\sim 1460$ days in long cadence mode (Jenkins et al. 2010). All the original light curves were processed and optimized for asteroseismic analysis following García et al. $(2011,2014)$, with the use of an inpainting algorithm (Mathur et al. 2010; Pires et al. 2015) to minimize the effect of up to 2 day-long gaps, during regular Earth downlinks and angular momentum dumps. A color-magnitude diagram for all the stars in the sample is shown in Fig. 1, which emphasizes the average difference in mass among the three open clusters (see also Corsaro et al. 2012 for more details about the general properties of the population of RGs in these open clusters). We notice that two stars marked as RGB, namely KIC 2437589 in NGC 6791 and KIC 5112361 in NGC 6819, are placed in the region of the color-magnitude diagram where the corresponding RC stars of the same clusters are located. Despite their peculiar location in the diagram, both stars have a RGB evolutionary stage unambiguously determined by their oscillations (Corsaro et al. 2012, 2017), with KIC 2437589 a possible evolved blue straggler (Brogaard et al. 2012; Corsaro et al. 2012) and KIC 5112361 a spectroscopic single lined binary (Milliman et al. 2014). 


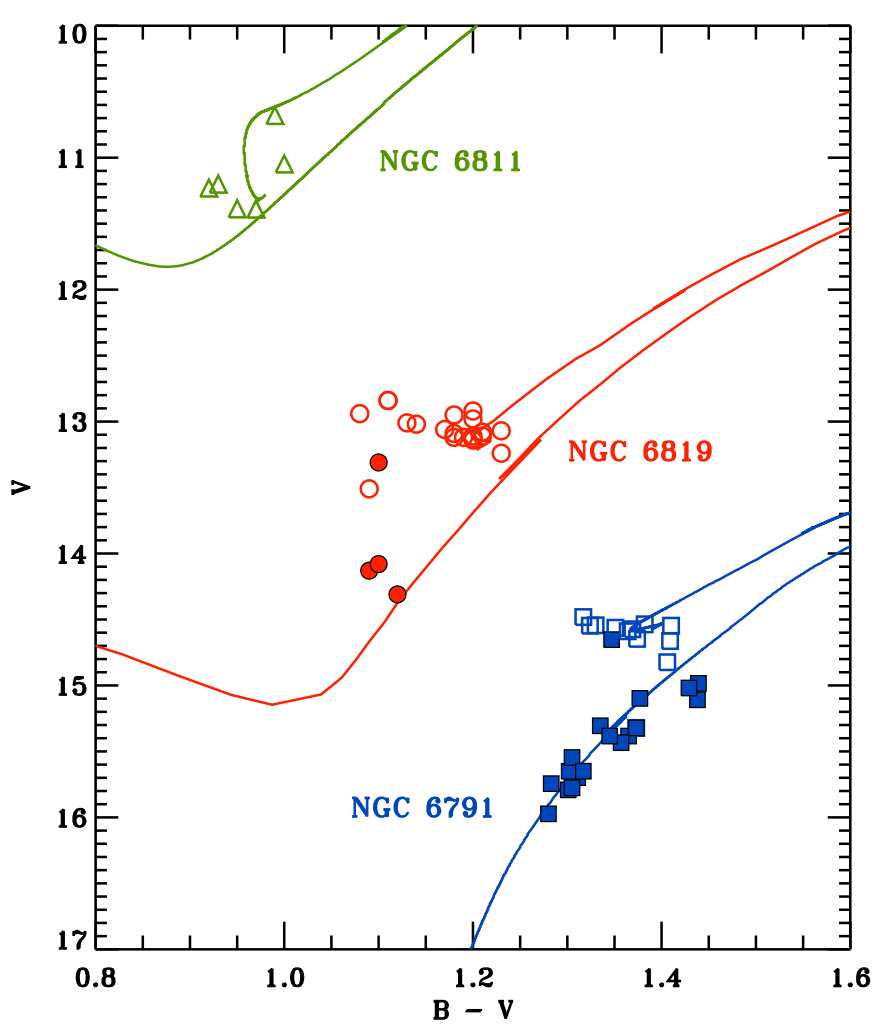

Fig. 1. Color-magnitude diagram of the three open clusters NGC 6791 (blue squares), NGC 6819 (red circles), and NGC 6811 (green triangles), with color and magnitudes of the 60 cluster RGs sourced from Stello et al. (2011), Corsaro et al. (2012). Hydrogen-shell-burning and core-He-burning RGs are shown with filled and open symbols, respectively, with an evolutionary stage identified according to Corsaro et al. (2012, 2017), and to Molenda-Żakowicz et al. (2014) for the two stars KIC 9776739 and KIC 9716090 . Isochrones are shown for each cluster as solid lines (see Stello et al. 2011, for more details).

\subsection{Effective temperatures}

For obtaining an accurate set of stellar effective temperatures for the entire sample of stars in this study we start from the revised KIC temperatures from the Sloan Digital Sky Survey (SDSS, Gunn et al. 2006) griz filters (Pinsonneault et al. 2012), which are available for all 60 targets. In addition, 36 stars (12 in NGC 6791, 20 in NGC 6819, and 4 in NGC 6811) have new temperatures determined from spectroscopy with ASPCAP (APOGEE Stellar Parameters and Chemical Abundances Pipeline, Zasowski et al. 2013; Nidever et al. 2015; Holtzman et al. 2015; García Pérez et al. 2016; Majewski et al. 2017), using the Data Release 13 (DR13, SDSS Collaboration 2016) of SDSS IV (Blanton et al. 2017), which includes the post-release metallicity correction (see Holtzman et al., in prep.). We therefore use ASPCAP temperatures, available from the latest release of the APOKASC catalog (Pinsonneault et al. 2014; Tayar et al. 2017), to apply a zero point shift to the temperatures from SDSS and correct them for the different cluster extinctions, which were based on the KIC map (Brown et al. 2011) in the work by Pinsonneault et al. (2012). In this way we put the temperatures from SDSS on the same scale as ASPCAP and we adopt the typical ASPCAP total temperature uncertainty (including both systematic and random effect) of $\sim 69 \mathrm{~K}$ as a reference (see Holtzman et al., in prep. and Tayar et al. 2017, for more discussion).
From a detailed comparison of individual temperature values, we noticed that several stars in NGC6791 (specifically KIC 2297384, KIC 2297825 on the RC, and KIC 2437270, KIC 2437589, KIC 2437972, KIC 2438038, KIC 2570094 on the RGB) have SDSS temperatures that are systematically cooler (by about $374 \mathrm{~K}$ ) than the average SDSS temperatures of the other red giants in the same cluster. This discrepancy is clearly visible by looking at the corresponding temperature differences shown with orange circles in Fig. 2, where the seven stars that we mentioned are marked by an asterisk. This $\sim 374 \mathrm{~K}$ offset is caused by an adopted reddening for the seven stars that is smaller than that of the other cluster stars by about 0.2 . For these seven stars we therefore decided to use $(V-K)$ color temperatures (and corresponding uncertainties of $110 \mathrm{~K}$, Hekker et al. 2011), which are available for all targets in NGC 6791. This choice is motivated by the fact that the $(V-K)$ color temperatures for the stars in NGC 6791 are in agreement (well within $1 \sigma$ ) with the ASPCAP temperatures from APOKASC and with those from our new temperature scale (see the comparison in Fig. 2). Finally, to avoid biasing our extinction correction applied to the SDSS temperature scale using the ASPCAP one, we remove KIC 2297384 and KIC 2297825 (the only two stars out of the seven with cool SDSS temperature that have also an ASPCAP temperature, see Fig. 2) from the computation of the zero point shift. The final temperature shifts that we obtain are $\left\langle T_{\text {eff,SDSS }}-T_{\text {eff,ASPCAP }}\right\rangle=282 \mathrm{~K}$ for NGC 6791, $173 \mathrm{~K}$ for NGC 6819, and 156 K for NGC 6811, showing that temperatures from SDSS photometry are systematically hotter than the ASPCAP ones (see Fig. 2). For simplicity, from here onwards the so-called SDSS-based temperature scale will refer to the temperatures from SDSS photometry corrected to the ASPCAP temperature scale as explained in this section, and supplemented with $(V-K)$ color temperatures adopted for the seven targets in NGC 6791 that show SDSS temperatures $400 \mathrm{~K}$ cooler than the other stars in the same cluster. We therefore adhere to the SDSS-based temperature scale to compute corrected mass estimates as discussed in Sect. 2.4. A complete list of the adopted temperatures for each star in the sample can be found in Tables A.1-A.3.

For completeness, we also note that $(V-K)$ color temperatures are available for all of the stars in NGC 6819 and in NGC 6811, except KIC 9776739 and KIC 9716090, which were studied by Molenda-Żakowicz et al. (2014) and have temperatures from spectroscopic data acquired from the Nordic Optical Telescope. As visible from Fig. 2, for the stars in NGC 6791 we find a good agreement between $(V-K)$ color temperatures and ASPCAP temperatures, while this agreement partially weakens for the stars in NGC 6819 where $(V-K)$ color temperatures are systematically cooler, and in NGC 6811 where instead they are hotter, although compatibility between the difference sources is still ensured within $1 \sigma$ in most cases. Lastly, the spectroscopic measurements from Molenda-Żakowicz et al. (2014) also agree (well within $1 \sigma$ ) with our SDSS-based temperatures for the same stars (Fig. 2).

\subsection{Metallicity}

We consider new cluster mean metallicities computed from ASPCAP corrected metallicities for the 36 targets for which they are available (see also Sect. 2.2, and Tayar et al. 2017). The metallicity values that we obtain for each cluster are listed in Table 1, with an uncertainty showing the standard deviation on the mean of the sample. Our estimates show that NGC 6791 has about twice the solar metallicity, NGC 6819 a close-to-solar metallicity, while NGC 6811 has a slightly sub-solar one (about 


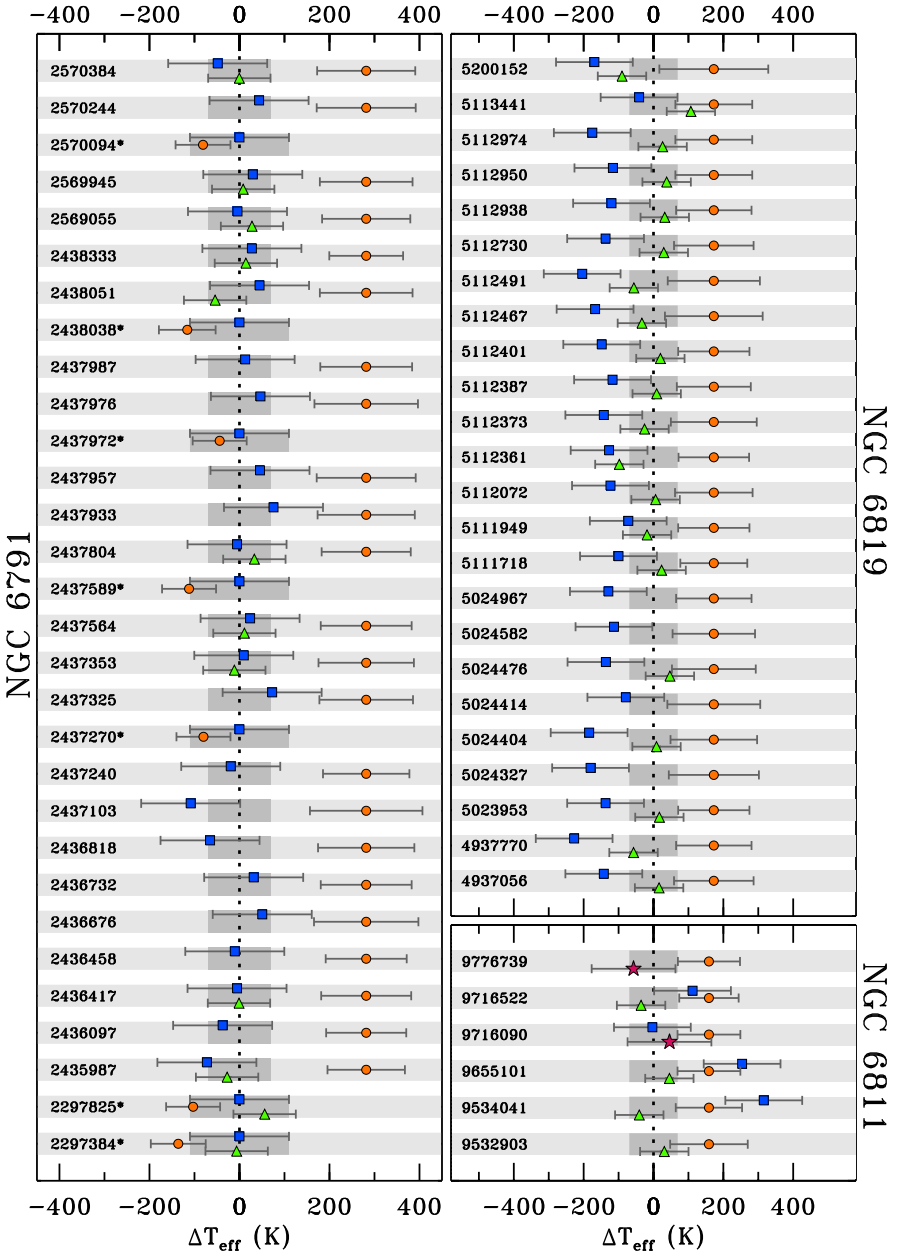

Fig. 2. Different sources for $T_{\text {eff }}$ as compared to the SDSS-based temperature scale prepared in this work and taken as a reference $\left(\Delta T_{\text {eff }}=\right.$ $0 \mathrm{~K}$ is marked with a dotted line), for all the 60 cluster RGs, labeled with their KIC ID. Orange circles refer to the original temperatures from the SDSS photometry (Pinsonneault et al. 2012), blue squares to $(V-K)$ color temperatures (Hekker et al. 2011), green triangles to ASPCAP temperatures from APOKASC (Pinsonneault et al. 2014), and purple stars to spectroscopic temperatures from Molenda-Żakowicz et al. (2014). The $1 \sigma$ uncertainties on each value are also overlaid. The dark gray shading around $\Delta T_{\text {eff }}=0$ delimits the $1 \sigma$ uncertainty adopted on the reference SDSS-based temperature scale. The seven stars of NGC 6791 that show cooler SDSS temperatures (see Sect. 2.2) are marked by an asterisk.

$20 \%$ less than that of the Sun). These cluster mean metallicities are in good agreement with previous estimates found in the literature for NGC 6791, $[\mathrm{Fe} / \mathrm{H}]=0.29 \pm 0.08$ (Brogaard et al. 2011), NGC 6819, $[\mathrm{Fe} / \mathrm{H}]=0.09 \pm 0.03$ (Bragaglia et al. 2001) and NGC 6811, $[\mathrm{Fe} / \mathrm{H}]=-0.040 \pm 0.002$ (Molenda-Żakowicz et al. 2014). We provide individual metallicity values from ASPCAP in Tables A.1-A.3, but we will consider only the cluster mean metallicities in the analysis presented in Sect. 5, to exploit the common origin that characterizes the stars in clusters. The metallicity range covered by the stars in the three clusters, about $\sim 0.4$ dex, while not large, is sufficient to identify the effects of metallicity on stellar granulation with high reliability and statistical evidence thanks to the homogeneity of the stellar properties shared by the members of each cluster (see also Sect. 7 for more discussion).
Table 1. Cluster mean metallicities and corresponding uncertainties as computed from the available ASPCAP metallicities for 36 stars of the sample investigated in this work (see also Tables A.1-A.3, for a list of all the values).

\begin{tabular}{|c|c|}
\hline Open cluster & $\begin{array}{c}\langle[\mathrm{Fe} / \mathrm{H}]\rangle_{\text {ASPCAP }} \\
(\operatorname{dex})\end{array}$ \\
\hline NGC 6791 & $0.32 \pm 0.02$ \\
\hline NGC 6819 & $0.04 \pm 0.03$ \\
\hline NGC 6811 & $-0.09 \pm 0.03$ \\
\hline
\end{tabular}

\subsection{Stellar mass}

We estimate the stellar masses and their uncertainties by basing our analysis on the asteroseismic scaling relations (e.g., Miglio et al. 2012). For RGs especially, it is recognized that the asteroseismic scaling relations have the tendency to overestimate masses because scaling relations are approximate in nature (e.g., Brown et al. 1991; Belkacem et al. 2011). To compensate for the overestimation, many authors have proposed different corrections, some empirically-based (Mosser et al. 2013), others resulting from calibrations using stellar evolution models (White et al. 2011; Miglio et al. 2012; Sharma et al. 2016; Guggenberger et al. 2016). In addition, Gaulme et al. (2016) compared masses and radii, obtained independently from both asteroseismic relations and dynamical modeling (eclipse photometry combined with radial velocities), of a sample of RGs in eclipsing binary systems. As a result, asteroseismic masses appear to be about 15\% larger than dynamical masses (see Gaulme et al. 2016, Fig. 9). However, since the sample studied by Gaulme et al. (2016) is rather small, we cannot infer how the mass correction depends on stellar parameters to provide a general correction law. Instead we follow Sharma et al. (2016) to compute a correction factor to the scaling relation of the large frequency separation $\Delta v$ (Ulrich 1986). This correction is based on a large grid of stellar evolution models. We therefore adopt a modified version of the standard scaling relation for mass, which reads as

$\frac{M}{M_{\odot}}=\left(\frac{v_{\text {max }}}{v_{\text {max }, \odot}}\right)^{3}\left(\frac{\Delta v}{\gamma \Delta v_{\odot}}\right)^{-4}\left(\frac{T_{\text {eff }}}{T_{\text {eff }, \odot}}\right)^{1.5}$,

where $\gamma$ is a correction factor for $\Delta v$, and is computed by taking into account the values of temperature, $v_{\max }$, and $\Delta v$ for each star, and the cluster mean metallicities from Table 1. Uncorrected mass estimates (from pure scaling) can easily be recovered with $M_{\text {uncorr }}=M \gamma^{-4}$. The value of $\Delta v$ for each star is computed from the frequencies of the three radial modes that are closest to $v_{\max }$. The frequencies are obtained from the peak bagging analysis performed by Corsaro et al. (in prep.), which consists in the fitting and identification of individual oscillation modes to extract their frequencies, amplitudes, and lifetimes. The peak bagging analysis for the cluster RGs of our sample is done following the same recipe presented by Corsaro et al. (2015; see also Corsaro et al. 2017), and by adopting the background parameters estimated in this work. We refer the reader to Corsaro et al. (2015) for a detailed description of the peakbagging analysis process using the Bayesian inference code DIAMONDS (Corsaro \& De Ridder 2014). The resulting stellar masses using the SDSS-based temperature scale (Sect. 2.2) and the cluster mean metallicities (Table 1) are listed in Tables A.1A.3, while the solar reference values $v_{\max , \odot}, \Delta v_{\odot}, T_{\text {eff, } \odot \text {, }}$ are presented in Sect. 3.2. By defining the mass difference between 


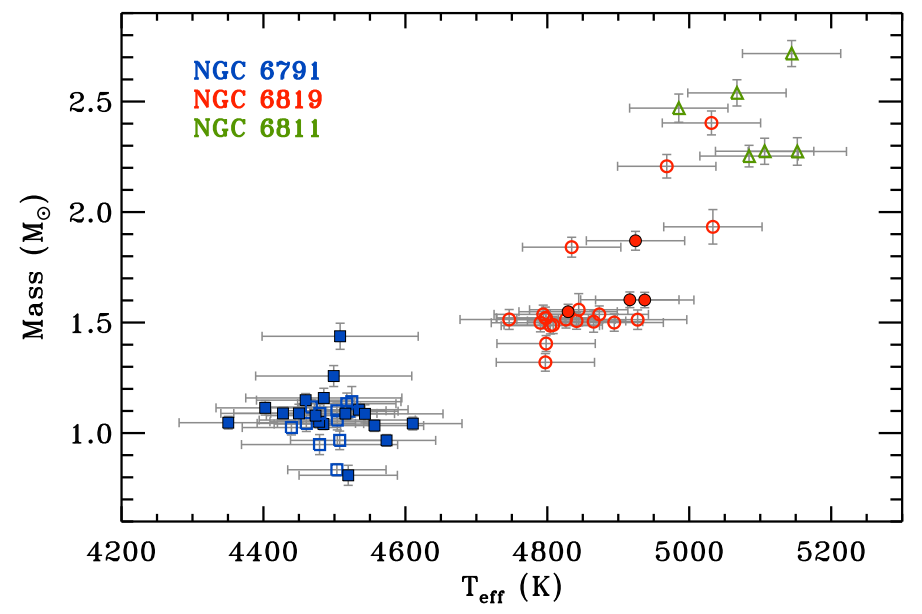

Fig. 3. Corrected stellar masses from Sect. 2.4, for the 60 cluster RGs, as a function of the temperatures from the SDSS-based temperature scale. The same symbol coding as in Fig. 1 is adopted. The $1 \sigma$ uncertainties in mass and $T_{\text {eff }}$ are also overlaid.

corrected and uncorrected estimates, $\Delta M=M-M_{\text {uncorr }}$, we note that the average mass correction $\left\langle\Delta M / M_{\text {uncorr }}\right\rangle$ is $-6.5 \%$ for NGC 6791, $0.5 \%$ for NGC 6819, and $4.0 \%$ for NGC 6811. In Fig. 3 we show our estimates of stellar mass as a function of $T_{\text {eff }}$ from the SDSS-based temperature scale, where a clear correlation between these two parameters is found, especially at temperatures higher than $4600 \mathrm{~K}$.

\section{Analysis of the background signal}

\subsection{Background fitting model}

The starting point of the analysis presented in this work is the measurement of the background properties observed in the stellar power spectral densities (PSDs) obtained from the Kepler light curves. We determine the parameters of the background signal (including granulation), as detailed in Corsaro et al. (2015), using the Bayesian inference code DiAMONDS (Corsaro \& De Ridder 2014). We adopt the background model presented by K14 (see also Kallinger et al. 2016), which can be expressed as

$P_{\mathrm{bkg}}(v)=N(v)+R(v)[B(v)+G(v)]$,

where we assume the noise component

$N(v)=W+\frac{2 \pi a_{\mathrm{n}}^{2} / b_{\mathrm{n}}}{1+\left(v / b_{\mathrm{n}}\right)^{2}}$,

to be the combination of a flat noise level $W$, mainly dominant at high frequency $(v \sim 200 \mu \mathrm{Hz})$, and a colored noise that can become significant at low frequency $(v \leq 20 \mu \mathrm{Hz})$, with $a_{\mathrm{n}}$ the amplitude, $b_{\mathrm{n}}$ its characteristic frequency, and $2 \pi$ a normalization constant (see also K14). The three super-Lorentzian components

$B(v)=\sum_{i=1}^{3} \frac{\zeta a_{i}^{2} / b_{i}}{1+\left(v / b_{i}\right)^{4}}$,

describe in decreasing frequency order, the granulation at frequencies close to $v_{\max }$, the meso-granulation for frequencies close to $v_{\max } / 3$, and a low-frequency component that incorporates heterogeneous signal coming from possible supergranulation and low-frequency variations in the time-series, whose analysis is beyond the scope of this work. Here $a_{i}$ is the rms intensity fluctuation (or amplitude), $b_{i}$ the characteristic frequency, and $\zeta=2 \sqrt{2} / \pi$ the normalization constant for a superLorentzian profile with its exponent set to four (see Karoff et al. 2013, K14 for more details). The power excess containing the stellar oscillations is modeled using a Gaussian envelope defined as

$G(v)=H_{\mathrm{osc}} \exp \left[-\frac{\left(v-v_{\max }\right)^{2}}{2 \sigma_{\mathrm{env}}^{2}}\right]$,

with $H_{\mathrm{osc}}$ the height of the oscillation bump and $\sigma_{\mathrm{env}}$ the standard deviation. Finally, all the components of the background signal, except those purely related to noise, $N(v)$, are modulated by the response function that corrects for the finite integration time of the long cadence Kepler observations, expressed as

$R(v)=\operatorname{sinc}^{2}\left(\frac{\pi v}{2 v_{\mathrm{Nyq}}}\right)$,

with $v_{\mathrm{Nyq}}=283.212 \mu \mathrm{Hz}$ the associated Nyquist frequency.

In Eq. (4), the meso-granulation component is associated with the parameters $\left(a_{2}, b_{2}\right)$, while the granulation component corresponds to the parameters $\left(a_{3}, b_{3}\right)$, with $a_{2}>a_{3}$ and $b_{2}<b_{3}$. The granulation component is the one that can be modeled through existing 3D hydrodynamical simulations of stellar atmospheres (e.g., see Trampedach et al. 1998; Ludwig \& Steffen 2016). However, in this work we will focus our analysis on the meso-granulation component, and we will refer to it from now on using the symbols $a_{\text {meso }} \equiv a_{2}$ and $b_{\text {meso }} \equiv b_{2}$. We will occasionally refer to the granulation component using the symbols $a_{\text {gran }} \equiv a_{3}$ and $b_{\text {gran }} \equiv b_{3}$. We have decided to select and analyze the meso-granulation component for the following reasons: (i) it is dominant over the granulation component both in height (PSD units) and in amplitude (e.g., see K14, Corsaro et al. 2015), so it is statistically more significant; (ii) it is well detached from the oscillation bump (with $v_{\max } \approx 3 b_{\text {meso }}$ ), hence less affected by biases and correlations associated with stellar oscillations than the granulation component, for which $v_{\max } \approx b_{\text {gran }}$ (e.g., see Corsaro \& De Ridder 2014); (iii) its characteristic parameters can be better determined than those of the granulation due to a higher signal-to-noise ratio, especially at high $v_{\max }$ (e.g., $>100 \mu \mathrm{Hz}$ ); (iv) it scales to surface gravity and temperature of the star similarly to the granulation component, because the meso-granulation represents a reorganization of the granulation at larger scales, so it originates from the same envelope convective motions. Hence, $a_{\text {meso }}$ and $b_{\text {meso }}$ can be used as accurate proxies for $a_{\text {gran }}$ and $b_{\text {gran }}$ (see K14). On average we find that $a_{\text {meso }} / a_{\text {gran }}=1.31 \pm 0.18$ and that $b_{\text {meso }} / b_{\text {gran }}=0.32 \pm 0.04$, throughout the $v_{\max }$ range spanned by our stellar sample.

Figure 4 shows an example of the resulting fit with DIAMONDS using the model given by Eq. (2) for the cluster RG KIC 4937056. The meso-granulation parameters and $v_{\max }$ from the fit to all the stars are presented in Figs. 5a and 6a, and listed in Tables B.1-B.3. We use uniform priors for all free parameters of the background model. The uniform prior boundaries are obtained by performing preliminary fits with the automated pipeline A2Z (Mathur et al. 2010), and using $v_{\max }$ values from Corsaro et al. (2012) and from Molenda-Żakowicz et al. (2014) as additional inputs. The configuring parameters of DIAMONDS that are adopted for the fitting of the background signal are provided in Appendix B. 


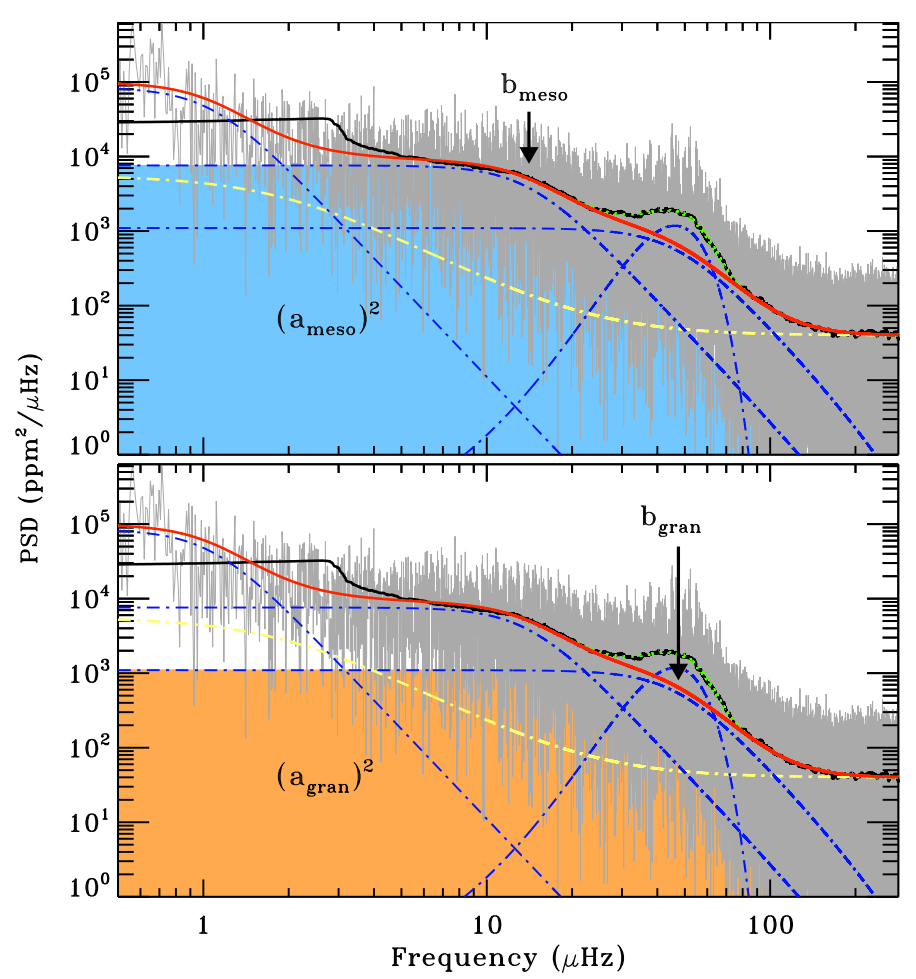

Fig. 4. Resulting background fit done with DiAmonDS (red curve) for the star KIC 4937056 in the cluster NGC 6819 overlaid on the original PSD of the star (in gray) and its smoothed version (black curve) using a boxcar with a width set to $\Delta v / 5$, where $\Delta v$ is computed using the $\Delta v-v_{\max }$ relation for RGs calibrated by Huber et al. (2011). The different components that constitute $B(v)$ (Eq. (4)) and $G(v)$ (Eq. (5)) are indicated with dot-dashed blue lines, while the noise term $N(v)$ (Eq. (3)) is shown with a dot-dashed yellow curve. The dotted green curve shows the overall fit of the background when the Gaussian envelope is included. Median values of the free parameters for each background component are used, as listed in Table B.2. Top panel: the meso-granulation component $\left(a_{\text {meso }}, b_{\text {meso }}\right)$ appears as a kink at $\sim 10 \mu \mathrm{Hz}$ (arrow) and its amplitude squared $\left(a_{\text {meso }}^{2}\right)$ is represented by the area of the shaded blue region. Bottom panel: same as the top panel but showing the parameters of the granulation component $\left(a_{\text {gran }}, b_{\text {gran }}\right)$.

\subsection{Solar reference values from VIRGO}

For a proper assessment of any metallicity dependence on granulation as presented in Sect. 4, we rely on our derivation of solar reference values. These reference values need to be as accurate and consistent as possible with the photometric data used for the cluster RGs presented in Sect. 2.1 and the analysis described in Sect. 3.1. For this purpose, we apply the background fitting approach with DIAMONDS (see Sect. 3.1) to the PSD of the Sun, using the background model defined by Eq. (2). We consider the combined light curves from VIRGO green and red channels $(g+r)$ to mimic the broad Kepler bandpass (Basri et al. 2010). We use an observing length coinciding with that of the Kepler light curves used in this work (see Sect. 2.1), thus obtaining the same frequency resolution in the resulting PSD. We consider two different combined light curves, the first one centered around the maximum of solar activity and the second one centered around the minimum. This is done to average out the effect of the solar activity cycle on the observed properties of the Sun. The two PSDs are then computed in the same way as for Kepler stars, and by re-binning to a sampling rate of $60 \mathrm{~s}$, close to that of the Kepler short cadence observation. We obtain two sets of solar parameters, one corresponding to maximum solar

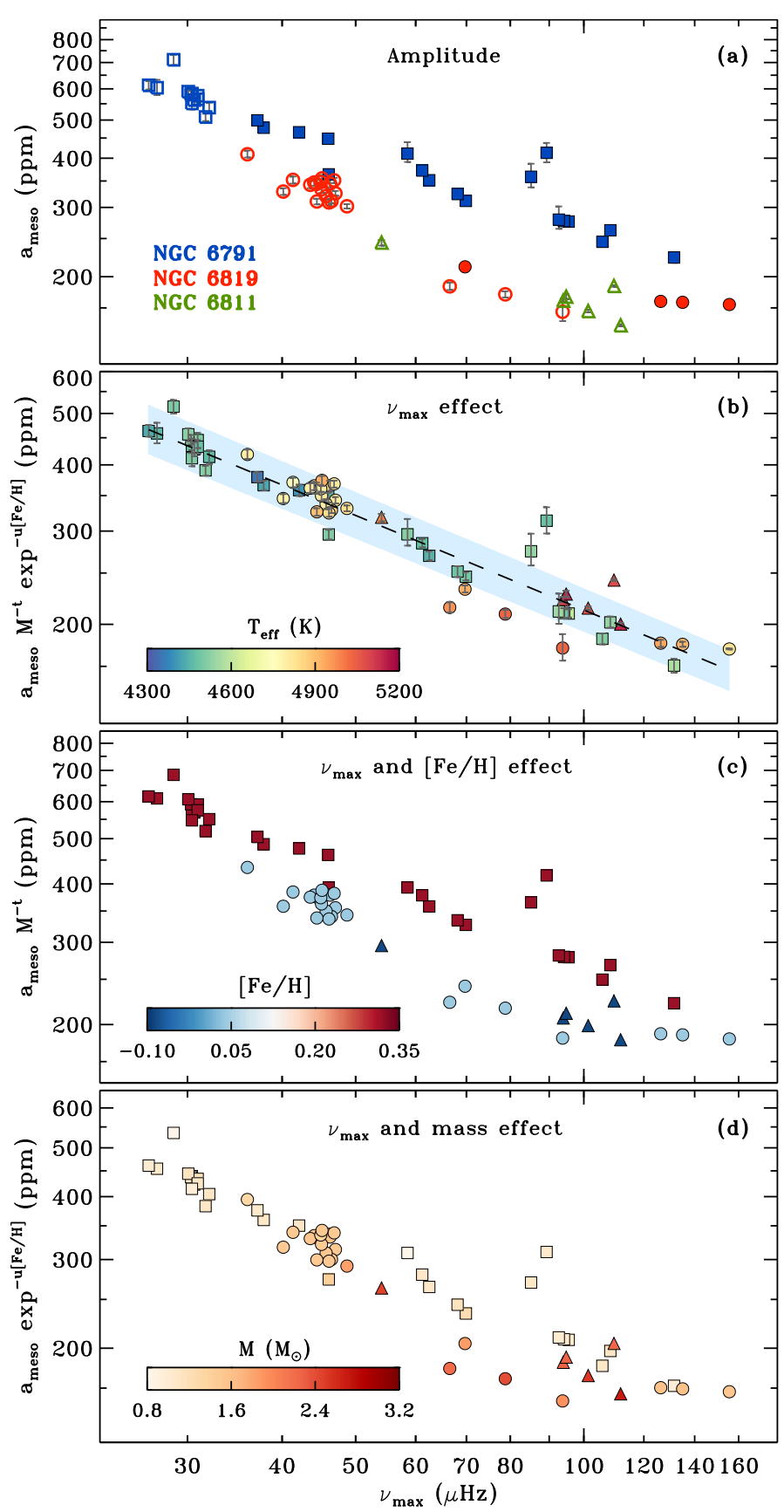

Fig. 5. Amplitude of the meso-granulation component as a function of $v_{\max }$ for the 60 cluster RGs. Color-coding in panel a shows the cluster membership, with open symbols for RC and filled ones for RGB stars, and the symbol types indicating the cluster membership as in Fig. 3. Panel $b$ shows the amplitudes after removing the effect of both mass and metallicity using the best scaling relation identified in Sect. 6 (model $\mathcal{M}_{a, 2}$ ), with the resulting fit marked by a dashed line and corresponding $1 \sigma$ credible region in shading, and with $T_{\text {eff }}$ from the SDSS-based temperature scale color-coded for each star. Panels $c$ and $d$ show the amplitudes after removing the effect of only mass and only metallicity, respectively, where cluster mean metallicities from ASPCAP and corrected masses of the stars from Sect. 2.4 are color-coded. Bayesian credible intervals of $68.3 \%$ on meso-granulation amplitudes are shown in panel $a$, and are rescaled in panel $b$.

activity and the other to minimum solar activity, which we average to obtain final estimates that are not biased by the activity cycle of the Sun. The final solar reference values are $a_{\text {meso, } \odot}=$ $56.0 \pm 0.2 \mathrm{ppm}$ (parts-per-milion) and $b_{\text {meso }, \odot}=752 \pm 3 \mu \mathrm{Hz}$ for 

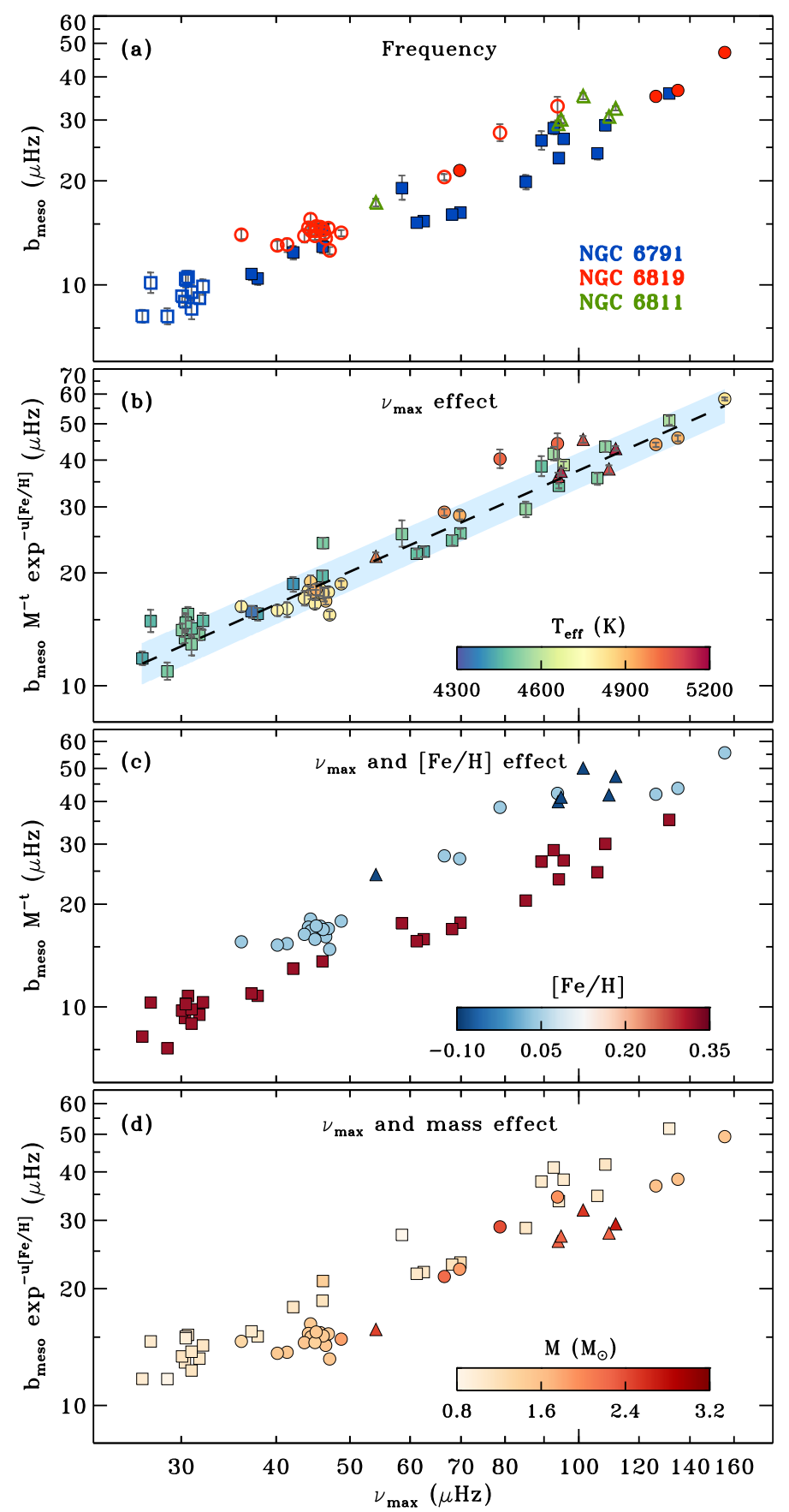

Fig. 6. Same as Fig. 5 but for the meso-granulation characteristic frequency.

the meso-granulation component, and $v_{\max , \odot}=3147 \pm 2 \mu \mathrm{Hz}$ for the power excess due to solar oscillations. We also include the large frequency separation as the average from the two datasets, $\Delta v_{\odot}=135.04 \pm 0.02 \mu \mathrm{Hz}$, whose calculation is explained in detail by Corsaro et al. (in prep.) and follows from a similar approach as that applied to the cluster RGs. Finally, the reference value for the solar effective temperature is the typical $T_{\text {eff }}=5777 \mathrm{~K}$ (e.g., Corsaro et al. 2013). Our estimates of $b_{\text {meso, } \odot}$ and $\nu_{\text {max, } \odot}$ agree within $1 \sigma$ and $2 \sigma$, respectively, with those obtained by K14. Our value of $a_{\text {meso, } \odot}$ is instead about 1.5 times larger than that of K14. We attribute this difference in $a_{\text {meso, } \odot}$ to the different preparation of the solar dataset, which in the case of K14 was accounting for 1-yr length observation of the VIRGO green channel only (centered at $550 \mathrm{~nm}$ ), and thus applying a simple linear transformation to obtain the reference amplitude at the central wavelength of the Kepler bandpass (664 nm).

\section{Scaling relations for granulation activity}

So far, empirical models related to the efficiency of the granulation signal, represented by the granulation amplitude $a_{\text {gran }}$ and its characteristic frequency $b_{\text {gran }}$ - or equivalently its time scale $\tau_{\text {gran }}=\left(2 \pi b_{\text {gran }}\right)^{-1}-$ have been investigated using large samples of stars with evolutionary stages ranging from the main sequence to the late RG phase (Mathur et al. 2011; Kallinger et al. 2016, K14). In the following we present the relevant scaling relations connecting $a_{\text {meso }}$ and $b_{\text {meso }}$ to $v_{\max }$, which in turn depends on stellar surface gravity and temperature, and mass of the stars, and for the first time we include the additional dependence on stellar metallicity.

\subsection{Meso-granulation amplitude $a_{\text {meso }}$}

As shown originally by Mathur et al. (2011), and later on by K14 and by Kallinger et al. (2016), the stellar granulation signal is strongly correlated with the atmospheric parameters of effective temperature and surface gravity, therefore $v_{\max }$ (Brown et al. 1991). For constant surface gravity, one can also test the effect of a varying stellar mass. In this work we consider a more general scaling relation of the form

$\left(\frac{a_{\text {meso }}}{a_{\text {meso }, \odot}}\right)=\beta\left(\frac{v_{\max }}{v_{\max , \odot}}\right)^{s}\left(\frac{M}{M_{\odot}}\right)^{t} \mathrm{e}^{u[\mathrm{Fe} / \mathrm{H}]}$,

where $M$ is the mass of the star, $[\mathrm{Fe} / \mathrm{H}]$ the metallicity, $s, t$, and $u$ are three exponents that need to be estimated, and $\beta$ is a scaling parameter for the solar reference values, typically set to $\beta=1$ (see also Corsaro et al. 2013, for more discussion about the implications of this scaling factor). We note that the solar values are only reference values used for the scalings, and a different choice of these values will not change either the quality of the fits or the Bayesian evidence associated with each model (see Sect. 5.1), but it would instead impact on the term $\beta$ that calibrates the scaling relation. As discussed in Sect. 3.2, we use our own solar reference values for consistency. We parametrize the metallicity with an exponential function because $[\mathrm{Fe} / \mathrm{H}]$ is already expressed in a logarithmic form and is compared to the solar value. For the study presented here, we consider two cases deriving from the generalized scaling relation of Eq. (7), one for $u=0$, which only accounts for $v_{\max }$ and mass dependencies, and one for $u \neq 0$, which also includes the metallicity effect on the amplitudes. We decide not to investigate the scaling relations incorporating only the dependency on $v_{\max }$ (obtained for $t=0$ and $u=0$ ) and only the dependency on $v_{\max }$ and $[\mathrm{Fe} / \mathrm{H}]$ (obtained for $t=0$ ), because the effect of a varying stellar mass in the amplitudes was already found to be significant from previous analyses (see K14).

In order to linearize the scaling relations and to be able to perform a thorough statistical analysis following the approach shown by Corsaro et al. (2013) and Bonanno et al. (2014), we apply the natural logarithm to Eq. (7), yielding

$\ln \left(\frac{a_{\text {meso }}}{a_{\text {meso } \odot \odot}}\right)=\ln \beta+s \ln \left(\frac{v_{\max }}{v_{\max , \odot}}\right)+t \ln \left(\frac{M}{M_{\odot}}\right)+u[\mathrm{Fe} / \mathrm{H}]$.

From here onwards, we identify the linearized scaling relations (or models) for the meso-granulation amplitude with the symbols $\mathcal{M}_{a, 1}$ for $u=0$, and $\mathcal{M}_{a, 2}$ for $u \neq 0$ (see Sect. 5 for more details). We also compute the analytical expressions for the uncertainties associated to the predicted meso-granulation amplitudes. 
Table 2. Median values of the inferred parameters $(s, t, u, \ln \beta)$ for all the models presented in Sect. 4, with the physical parameter they relate to shown in brackets.

\begin{tabular}{cccccc}
\hline \hline Model & $s\left(v_{\max }\right)$ & $t(M)$ & $u([\mathrm{Fe} / \mathrm{H}])$ & $\ln \beta$ & $\Lambda_{\max }$ \\
\hline $\mathcal{M}_{a, 1}$ & $-0.550_{-0.009}^{+0.008}$ & $-0.67_{-0.02}^{+0.02}$ & - & $-0.25_{-0.04}^{+0.04}$ & -46.4 \\
$\mathcal{M}_{a, 2}$ & $-0.593_{-0.010}^{+0.010}$ & $-0.21_{-0.05}^{+0.04}$ & $0.89_{-0.08}^{+0.08}$ & $-0.71_{-0.06}^{+0.06}$ & -31.3 \\
\hline $\mathcal{M}_{b, 1}$ & $0.954_{-0.008}^{+0.007}$ & - & - & $0.03_{-0.03}^{+0.03}$ & -227.4 \\
$\mathcal{M}_{b, 2}$ & $0.917_{-0.009}^{+0.008}$ & $0.20_{-0.02}^{+0.02}$ & - & $-0.19_{-0.03}^{+0.03}$ & -120.2 \\
$\mathcal{M}_{b, 3}$ & $0.889_{-0.009}^{+0.009}$ & - & $-0.52_{-0.03}^{+0.03}$ & $-0.16_{-0.03}^{+0.03}$ & -37.1 \\
$\mathcal{M}_{b, 4}$ & $0.898_{-0.014}^{+0.012}$ & $-0.38_{-0.06}^{+0.06}$ & $-1.15_{-0.10}^{+0.12}$ & $0.10_{-0.07}^{+0.06}$ & -2.5 \\
\hline
\end{tabular}

Notes. Bayesian credible intervals of $68.3 \%$ are added. The maximum value for the log-likelihood function, $\Lambda_{\max }$, is reported as fit quality indicator, where a larger value corresponds to a better fit to the observations.

According to a standard Gaussian error propagation (see also Corsaro et al. 2013), we obtain the total relative uncertainties

$\widetilde{\sigma}_{a}^{2}(s, t, u)=\widetilde{\sigma}_{a_{\text {meso }}}^{2}+s^{2} \widetilde{\sigma}_{v_{\max }}^{2}+t^{2} \widetilde{\sigma}_{M}^{2}+u^{2} \widetilde{\sigma}_{[\mathrm{Fe} / \mathrm{H}]}^{2}$,

which clearly depend upon the free parameters of the corresponding scaling relations, except for the offset term $\ln \beta$ that is not directly depending on any of the observables in this formulation. The relative uncertainties are $\widetilde{\sigma}_{a_{\text {meso }}} \equiv \sigma_{a_{\text {meso }}} / a_{\text {meso }}$, $\widetilde{\sigma}_{v_{\max }} \equiv \sigma_{v_{\max }} / v_{\max }, \widetilde{\sigma}_{M} \equiv \sigma_{M} / M$, while $\widetilde{\sigma}_{[\mathrm{Fe} / \mathrm{H}]}$ is the formal uncertainty on metallicity (which is already in relative units). Clearly, the total relative uncertainty for amplitude predictions from model $\mathcal{M}_{a, 1}$ is obtained by imposing $u=0$, while that from model $\mathcal{M}_{a, 2}$ is the general form with $u \neq 0$. These parameterdependent uncertainties, and the linearized models, will be used for the Bayesian inference described in Sect. 5.

\subsection{Meso-granulation characteristic frequency $b_{\text {meso }}$}

The frequency scale of the granulation signal is known to follow a tight scaling with the stellar surface gravity, like the amplitude. In particular Mathur et al. (2011) showed that the time scale of the granulation signal, $\tau_{\text {gran }}$, scales with $v_{\text {max }}$. This result was later on confirmed by K14. For our analysis we adopt a generalized scaling relation for the characteristic frequency of the meso-granulation signal, of the form

$\left(\frac{b_{\text {meso }}}{b_{\text {meso } \odot}}\right)=\beta\left(\frac{v_{\max }}{v_{\max , \odot}}\right)^{s}\left(\frac{M}{M_{\odot}}\right)^{t} \mathrm{e}^{u[\mathrm{Fe} / \mathrm{H}]}$,

with $\beta$ once again a scaling factor and $s, t, u$ the exponents that need to be estimated. For this property of the meso-granulation we consider the linearized form of Eq. (10), and test four different models, which we label as $\mathcal{M}_{b, 1}$ for $t=0$ and $u=0, \mathcal{M}_{b, 2}$ for $u=0, \mathcal{M}_{b, 3}$ for $t=0$, and $\mathcal{M}_{b, 4}$ for $t \neq 0$ and $u \neq 0$. In this formulation, model $\mathcal{M}_{b, 1}$ is clearly the simplest, not including both mass and metallicity terms, while models $\mathcal{M}_{b, 2}$ and $\mathcal{M}_{b, 3}$ consider the dependence on mass and metallicity separately from one another and are equally complex in terms of parameters. Model $\mathcal{M}_{b, 4}$ is instead the most generalized one, where both mass and metallicity effects are included at the same time.

Following the same approach used for the meso-granulation amplitude, we derive the analytical expressions for the parameter-dependent relative uncertainties associated with the predicted meso-granulation frequencies, yielding the general form

$\widetilde{\sigma}_{b}^{2}(s, t, u)=\widetilde{\sigma}_{b_{\text {meso }}}^{2}+s^{2} \widetilde{\sigma}_{v_{\max }}^{2}+t^{2} \widetilde{\sigma}_{M}^{2}+u^{2} \widetilde{\sigma}_{[\mathrm{Fe} / \mathrm{H}]}^{2}$, with the same definitions as in Sect. 4.1 for $v_{\max }, M$, and $[\mathrm{Fe} / \mathrm{H}]$, and with $\widetilde{\sigma}_{b_{\text {meso }}} \equiv \sigma_{b_{\text {meso }}} / b_{\text {meso }}$. Like for the amplitudes, we can obtain the total relative uncertainty for the frequency predictions by imposing $t=0$ and $u=0$ for model $\mathcal{M}_{b, 1}, u=0$ for model $\mathcal{M}_{b, 2}, t=0$ for model $\mathcal{M}_{b, 3}$, and $t \neq 0$ and $u \neq 0$ for model $\mathcal{M}_{b, 4}$.

\section{Bayesian inference}

We perform a Bayesian inference on the models presented in Sect. 4 by adopting a Gaussian likelihood where the residuals, assumed to be Gaussian distributed, arise from the difference between the observed and predicted natural logarithms of the parameters that describe the granulation activity. The Gaussian loglikelihood, similar to Corsaro et al. (2013) and Bonanno et al. (2014), therefore reads

$\Lambda(\boldsymbol{\theta})=\Lambda_{0}(\boldsymbol{\theta})-\frac{1}{2} \sum_{i=1}^{N}\left[\frac{\Delta_{i}(\boldsymbol{\theta})}{\widetilde{\sigma}_{i}(\boldsymbol{\theta})}\right]^{2}$,

where $\boldsymbol{\theta}$ is the parameter vector, for example $(\beta, s, t, u)$ for the model $\mathcal{M}_{a, 2}, N$ is the total number of stars, and $\Lambda_{0}(\boldsymbol{\theta})$ is a term depending on the relative uncertainties, given by

$\Lambda_{0}(\boldsymbol{\theta})=-\sum_{i=1}^{N} \ln \sqrt{2 \pi} \widetilde{\sigma}_{i}(\boldsymbol{\theta})$.

The residuals between observed and predicted values are defined as

$\Delta_{i}(\boldsymbol{\theta})=\ln a_{\text {meso }}^{\mathrm{obs}}-\ln a_{\text {meso }}^{\mathrm{pred}}(\boldsymbol{\theta})$.

The results from the Bayesian parameter estimation are listed in Table 2, with $\Lambda_{\max }$ representing the maximum value of the loglikelihood function (Eq. (12)), increasing as the fit to the data improves. We note that in order to evaluate whether the fit quality of a model is better than that of other models, $\Lambda_{\max }$ has to be compared to that of a competitor model and has therefore no meaning on its own. A thorough assessment of the statistical significance of a model is presented in Sect. 5.1. The predictions obtained from the estimated parameters are compared to the observations in Figs. C. 1 and C. 2 for all the models considered in Sect 4. 
E. Corsaro et al.: Metallicity effect on stellar granulation from cluster red giants

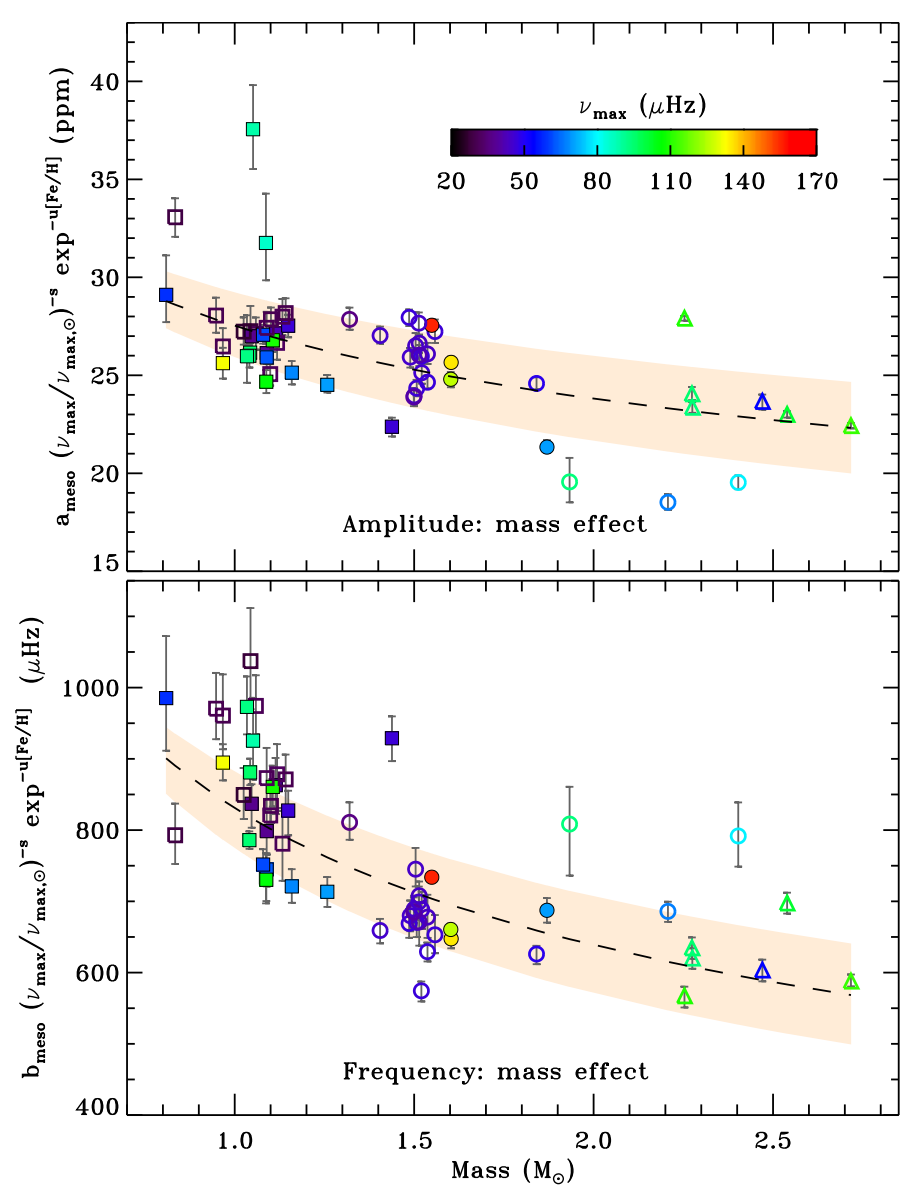

Fig. 7. Amplitudes (top panel) and frequencies (bottom panel) of the meso-granulation component for the 60 cluster RGs, as a function of the corrected stellar masses from Sect. 2.4. Dependencies upon $v_{\max }$ and $[\mathrm{Fe} / \mathrm{H}]$ were removed by means of the best scaling relations identified in Sect. 5.1 (Eq. (7) for the top panel and Eq. (10) for the bottom panel). The dashed lines mark the fits from the best scaling relations, while the shaded regions delimit the $1 \sigma$ credible regions from the estimated parameters (Table 2). Open symbols denote RC stars, while filled symbols are RGB stars, with cluster membership indicated by the same symbol types as in Fig. 3. The values of $v_{\max }$ are color-coded for each star. Rescaled Bayesian credible intervals of $68.3 \%$ for each mesogranulation parameter are overlaid on both panels.

\subsection{Model hypothesis testing}

The Bayesian model hypothesis test is performed by computing the so-called odds ratio between two competing models $\mathcal{M}_{i}$ and $\mathcal{M}_{j}$

$O_{i j}=\frac{\mathcal{E}_{i}}{\mathcal{E}_{j}} \frac{\pi\left(\mathcal{M}_{i}\right)}{\pi\left(\mathcal{M}_{j}\right)}=\mathcal{B}_{i j} \frac{\pi\left(\mathcal{M}_{i}\right)}{\pi\left(\mathcal{M}_{j}\right)}$,

where $\mathcal{B}_{i j}$ is the Bayes factor given as the ratio of the Bayesian evidences $(\mathcal{E})$ of the two models, and $\pi(\mathcal{M})$ is our model prior, or equivalently model weight, assigned to each of the models investigated. Given the linearity of the models, model priors for multiplicity adjustment can be taken into account (Scott \& Berger 2010). For this purpose, we consider the model prior function proposed by Scott \& Berger (2010), which for a model having $k$ free parameters out of a full set of $m$ free parameters investigated, i. e. the total number of parameters to test, reads as

$\pi\left(\mathcal{M}^{k}\right)=\frac{k !(m-k) !}{m !(m+1)}$.

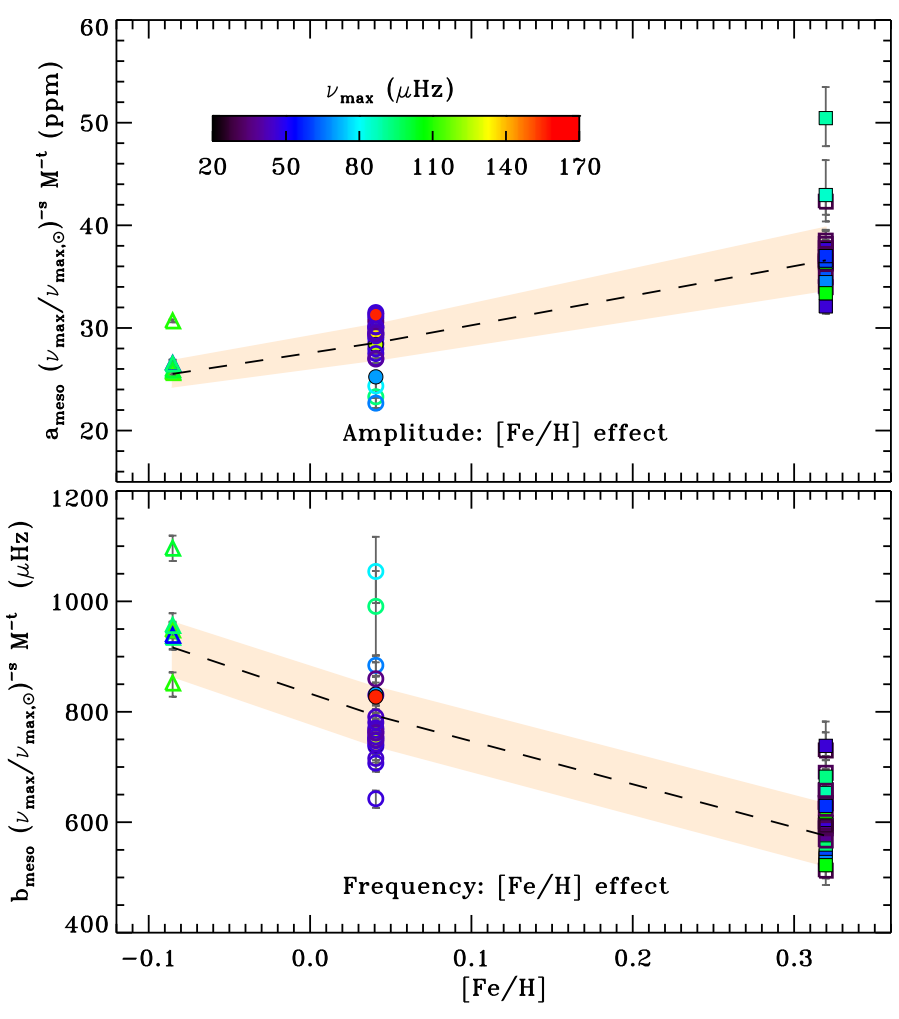

Fig. 8. Same as Fig. 7 but showing the meso-granulation parameters with the $v_{\max }$ and stellar mass dependencies removed, as a function of the cluster mean metallicities computed from ASPCAP (Sect. 2.3).

In our analysis, the linearized models for amplitudes (see Sect. 4.1) and characteristic frequency (see Sect. 4.2) account for a total of $m=3$ free parameters $(s, t, u)$ related to the observables. The intercept $\ln \beta$ is not included in the count of free parameters relevant for the model prior because a model with the intercept as the only free parameter is the null model, with $k=0$. The model priors give $\pi\left(\mathcal{M}^{\mathrm{k}=3}\right) / \pi\left(\mathcal{M}^{\mathrm{k}=2}\right)=$ $\pi\left(\mathcal{M}^{\mathrm{k}=3}\right) / \pi\left(\mathcal{M}^{\mathrm{k}=1}\right)=3$.

We compute the Bayes factor for each pair of scaling relations following Corsaro et al. (2013). Since the Bayesian evidence of a model taken singularly is not meaningful, the best (or statistically more likely) model is chosen as the one that maximizes the odds ratio given by Eq. (15) in a comparison between pairs of models, for all the models considered in the analysis. Results for the model comparison for all the models investigated in this work are presented in Table 3 for $a_{\text {meso }}$ and $b_{\text {meso }}$, with the most favored models highlighted with shades. The net effects caused by stellar mass and metallicity on the meso-granulation properties can be isolated by adopting the most favored scaling relations selected by our model comparison process. The results are depicted in Figs. $5 \mathrm{~b}$ and $6 \mathrm{~b}$, both as a function of $v_{\max }$, and in Figs. 7 and 8 as a function of mass and metallicity, respectively.

\section{Results}

From our inference on the models presented in Sect. 4, stellar mass and especially metallicity appear to play a statistically significant role in the meso-granulation properties of the stars. In Figs. 5a and c, the latter showing the amplitudes after the mass effect has been removed, we observe two distinct groups of stars. The first group corresponds to stars with supersolar metallicity and high meso-granulation amplitudes (from NGC 6791), while the second one is composed of stars with 
Table 3. Natural logarithms of the odds ratio, $\ln O_{i j}=\ln \pi\left(\mathcal{M}_{i}\right)-$ $\ln \pi\left(\mathcal{M}_{j}\right)+\ln \mathcal{E}_{i}-\ln \mathcal{E}_{j}$, for each pair of models $\left(\mathcal{M}_{i}, \mathcal{M}_{j}\right.$ related to the meso-granulation amplitude $a_{\text {meso }}$ (marked with subscript $a$ ), and characteristic frequency $b_{\text {meso }}$ (marked with subscript $b$ ).

\begin{tabular}{cccccc}
\hline \hline Model & $\mathcal{M}_{a, 1}$ & $\mathcal{M}_{a, 2}$ & $\mathcal{M}_{b, 1}$ & $\mathcal{M}_{b, 2}$ & $\mathcal{M}_{b, 3}$ \\
\hline $\mathcal{M}_{a, 1}$ & - & & & & \\
$\mathcal{M}_{a, 2}$ & 15.5 & - & & & \\
\hline $\mathcal{M}_{b, 1}$ & & & - & & \\
$\mathcal{M}_{b, 2}$ & & & 108.0 & - & \\
$\mathcal{M}_{b, 3}$ & & & 191.9 & 83.9 & - \\
$\mathcal{M}_{b, 4}$ & & & 225.8 & 117.8 & 33.9 \\
\hline
\end{tabular}

Notes. Shading indicates the most likely models $\left(\mathcal{M}_{a, 2}\right.$ for $a_{\text {meso }}$ and $\mathcal{M}_{b, 4}$ for $b_{\text {meso }}$ ), meaning that they maximize all the odds ratios when compared to the competitor models.

close-to-solar metallicity and low meso-granulation amplitudes (from NGC 6819 and NGC 6811). We observe these two groups independently of whether a star is RC or RGB. The trend with metallicity is also clearly shown in the top panel of Fig. 8, where the amplitudes have been corrected for $v_{\max }$ and mass dependencies.

In Figs. 6a and c, the latter showing meso-granulation frequencies without the mass effect, we see that the stars belonging to NGC 6791 have the tendency to exhibit frequencies smaller than the stars of the other two clusters. This is more evident from the bottom panel of Fig. 8, in which the meso-granulation frequencies were rescaled to remove the effect of a varying $v_{\max }$ and stellar mass. These observational considerations are reflected in the values of the exponents of the scaling relations and in our model hypothesis testing, which we discuss in Sects. 6.1 and 6.2.

From both Figs. 5a and 6a we note that the typical $v_{\max }$ of the RC stars within a cluster changes significantly from one cluster to another, with $v_{\max } \sim 20 \mu \mathrm{Hz}$ for NGC 6791, $v_{\max } \sim 35 \mu \mathrm{Hz}$ for NGC 6819 , and $v_{\max } \sim 100 \mu \mathrm{Hz}$ for NGC 6811 . This difference is mainly caused by the different average masses of the RC stars in each cluster (Fig. 3) because for a constant stellar radius $v_{\max } \propto M T_{\text {eff }}^{-0.5}$ (see also the color-magnitude diagram shown in Fig. 1 and those presented in Fig. 9 of Corsaro et al. 2012). A detailed discussion of our findings can be found in Sect. 7, where we highlight their implications and physical interpretations.

\subsection{Meso-granulation amplitude $a_{\text {meso }}$}

We find that the scaling relations for $a_{\text {meso }}$ have a negative exponent $t$, for stellar mass, of -0.67 and -0.21 (models $\mathcal{M}_{a, 1}$ and $\mathcal{M}_{a, 2}$ respectively), and a positive exponent $u$, for $[\mathrm{Fe} / \mathrm{H}]$, of about 0.89 (model $\mathcal{M}_{a, 2}$ ). The results from the parameter estimation of the best model $\mathcal{M}_{a, 2}$ imply that the dependency on metallicity is more than four times stronger than that on stellar mass, and that an increasing metallicity increases the amplitude of the signal (see Fig. 5c and our fit marked in Fig. 8, top panel). If we consider stars at constant surface gravity - to first approximation at constant $v_{\max }$ given that the temperature range of our sample of stars is not large $\left(\sim 10^{3} \mathrm{~K}\right)$ - the effect of metallicity is opposite to that of stellar mass (Fig. 5d versus Fig. 5c, and Fig. 7 versus Fig. 8, top panels).

The dependency on metallicity estimated from the best model $\mathcal{M}_{a, 2}$ is about 1.5 times stronger than the dependency on $v_{\max }$, hence than on $g / \sqrt{T_{\text {eff }}}$ and to first approximation on surface gravity, with an exponent $s$ set around -0.55 and -0.59 for models $\mathcal{M}_{a, 1}$ and $\mathcal{M}_{a, 2}$, respectively. The exponent $s$ is close, although not compatible within the quoted errors, to the value of -0.61 found by K14 using a larger sample of field stars that also included main sequence stars. The exponent $t$ of model $\mathcal{M}_{a, 2}$, including $[\mathrm{Fe} / \mathrm{H}]$, is also compatible (within $1 \sigma$ ) with that found by $\mathrm{K} 14,-0.26$, while model $\mathcal{M}_{a, 1}$ has a three times larger estimate of $t$ with respect to that of model $\mathcal{M}_{a, 2}$. The stronger dependency on stellar mass in the scaling relation associated to model $\mathcal{M}_{a, 1}$, as compared to that of $\mathcal{M}_{a, 2}$, is also a consequence of the lack of a term that takes into account the different stellar metallicity, which is significantly different in NGC 6791 with respect to NGC 6811 and NGC 6811 (a factor of about two). Differences between our exponents and those from the literature also rely on: (i) the adoption of a sample of only RGs (K14 included both field RGs and main sequence stars), hence of a range of surface gravities $(2.3 \leq \log g \leq 3.1)$ and temperatures $\left(4350 \mathrm{~K}<T_{\text {eff }}<5150 \mathrm{~K}\right)$ typical of evolved low- and intermediate-mass stars; (ii) the use of different data sources and of corrected stellar masses (as derived in Sect. 2.4); (iii) the use of a more accurate and uniform set of fundamental stellar properties, stemming from the cluster membership of the targets.

In regard to the solar reference values used in this work (Sect. 3.2), our estimation of the proportionality term $\beta$ suggests that the reference amplitude for our sample should be smaller

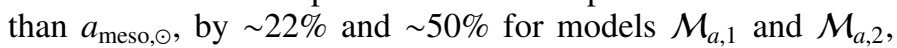
respectively. This probably signals a break-down of linearity across the orders of magnitude in surface gravity, that separate our sample of RGs from the Sun.

As shown in Table 3, the Bayesian model comparison largely favors model $\mathcal{M}_{a, 2}$, including the metallicity term, against $\mathcal{M}_{a, 1}$ because the corresponding odds ratio $\left(\ln O_{2,1} \simeq 16\right)$ is well above a strong evidence condition $\left(\ln O_{i j}>5\right.$ for model $\mathcal{M}_{i}$ versus model $\mathcal{M}_{j}$, according to the Jeffreys' scale of strength), thus justifying the inclusion of an additional dependency on metallicity. This is also observed in the much higher maximum likelihood value of $\mathcal{M}_{a, 2}$ compared to that of $\mathcal{M}_{a, 1}$, with $\Lambda_{\max }^{a, 2}-\Lambda_{\max }^{a, 1} \simeq 15$ (see Table 2). We therefore recommend the adoption of the scaling relation given by Eq. (7) when predicting meso-granulation amplitudes for RGs having $20 \mu \mathrm{Hz}<v_{\max }<160 \mu \mathrm{Hz}$. Although for solar metallicity stars, $[\mathrm{Fe} / \mathrm{H}]=0$, model $\mathcal{M}_{a, 2}$ formally reduces to the analytical form of $\mathcal{M}_{a, 1}$, the exponent for the stellar metallicity is $u \neq 0$ and for the exponents $s$ (for $v_{\max }$ ) and $t$ (for stellar mass) of the scaling relation estimates from model $\mathcal{M}_{a, 2}$, as listed in Table 2, should still be taken into account. Finally, as seen from Fig. C.1, and also apparent in Fig. 5b where we show the amplitudes corrected for mass and metallicity effects, we do not observe any clear difference between RC and RGB stars because the residuals from our predictions are on the same level for both evolutionary stages (on average around $8 \%$ ).

\subsection{Meso-granulation characteristic frequency $b_{\text {meso }}$}

For the characteristic frequency of the meso-granulation we have tested the four different models described in Sect. 4.2. In this case, an increasing metallicity appears to reduce $b_{\text {meso }}$, namely to increase the time scale of the meso-granulation. This can be seen from Fig. 6a, and more so from Fig. 6c, where the mass effect has been removed, and from the bottom panel of Fig. 8, in which the trend with metallicity has been isolated from the strong dependency on $v_{\max }$. This result is confirmed by the exponents estimated for Eq. (10). We find that the exponent related to metallicity, $u$, is -0.52 for model $\mathcal{M}_{b, 3}$ and -1.15 for the best 
model $\mathcal{M}_{b, 4}$, indicating that the strength of the relation between $b_{\text {meso }}$ and $[\mathrm{Fe} / \mathrm{H}]$ is comparable to that between $b_{\text {meso }}$ and $v_{\max }$.

An exponent $s \simeq 0.9$, which is found for all the models for $b_{\text {meso }}$ tested in this work, shows that $b_{\text {meso }}$ and $v_{\max }$ are almost linearly related, implying that the two parameters do follow a similar scaling (see Sect. 7 for a discussion on this result). The $s$ exponent found in this work is on average only $5 \%$ smaller, although significant according to the quoted errors, than that obtained by K14 using a similar scaling relation for the same mesogranulation component. Once again we attribute this difference to the different sample, range of fundamental stellar properties, and sources of observational data used.

According to our estimates of the proportionality term $\beta$, we find that an optimal reference value would range from $\sim 83 \%$ (model $\mathcal{M}_{b, 2}$ ), up to $\sim 110 \%$ of $b_{\text {meso, } \odot}\left(\right.$ model $\mathcal{M}_{b, 4}$ ). Overall, this is closer to the adopted solar value than what was found in the case of the meso-granulation amplitude, and the difference from unity in the parameter $\beta$ is not even statistically significant for model $\mathcal{M}_{b, 1}$, and only marginally significant for model $\mathcal{M}_{b, 4}$ (within $2 \sigma$ ). This suggests that a possible break-down in linearity for the characteristic timescale of the granulation and meso-granulation signals between our sample and the Sun is in general less likely than for amplitudes.

The effect of mass on $b_{\text {meso }}$, after surface gravity has been accounted for, is in the same direction as that of metallicity (Fig. 6d versus Fig. 6c, and Fig. 7 versus Fig. 8, bottom panels). The associated exponent $t$ reaches up to only -0.38 for the best model $\mathcal{M}_{b, 4}$. This weak mass dependence is also evident from the Bayesian model comparison (Table 3) where model $\mathcal{M}_{b, 3}$, incorporating only metallicity and $v_{\max }$ dependencies, far exceeds the strong evidence condition against model $\mathcal{M}_{b, 2}\left(\ln O_{3,2} \simeq 84\right)$, the latter including only stellar mass and $v_{\max }$. However, $\mathcal{M}_{b, 4}$, which encompasses both mass and $[\mathrm{Fe} / \mathrm{H}]$ on top of $v_{\max }$, is significantly better than a model that incorporates one or the other $\left(\ln O_{4,2} \simeq 118, \ln O_{4,3} \simeq 34\right)$. We note that the odds ratio between model $\mathcal{M}_{b, 4}$ and model $\mathcal{M}_{b, 3}$ is much smaller than that between $\mathcal{M}_{b, 3}$ and $\mathcal{M}_{b, 2}$, confirming that even in this case the stellar mass does not constitute a dominant contribution to $b_{\text {meso }}$. Like $a_{\text {meso, }}$, we recommend the adoption of Eq. (10) for predictions of the meso-granulation characteristic frequency for RGs in the $v_{\max }$ range investigated. We show the fit results in Fig. C.2, where the scatter in the residuals is on average around $8-9 \%$ for the different models, and in Fig. $6 \mathrm{~b}$ where we correct for mass and metallicity effects. Similar to the case of $a_{\text {meso }}$, we do not find any evidence for a systematic difference between RC and RGB stars of the same cluster.

\subsection{Assessing the reliability of the metallicity effect}

To further validate our results we perform three additional analyses described below. First, to make sure no biases are caused by the numerical method implemented in DIAMONDS, we derive the background parameters discussed in Sect. 3.1 using another automated fitting routine, based on a Bayesian maximum a posteriori method (Gaulme et al. 2009). We find that the resulting values of $v_{\max }, a_{\text {meso }}$, and $b_{\text {meso }}$ agree with the measurements derived with DIAMONDS on average within 1,3 , and $6 \%$, respectively, compatible within the Bayesian credible intervals.

Second, we measure the granulation flicker, $F_{8}$, introduced by Bastien et al. (2013), from the Kepler light curves for the stars in our sample satisfying the limits of applicability defined in Bastien et al. (2016, a total of 26 targets, 3 from NGC 6819, 4 from NGC 6811, and 19 from NGC 6819). For each of these stars we therefore have the amplitude in parts-per-thousand (ppt)

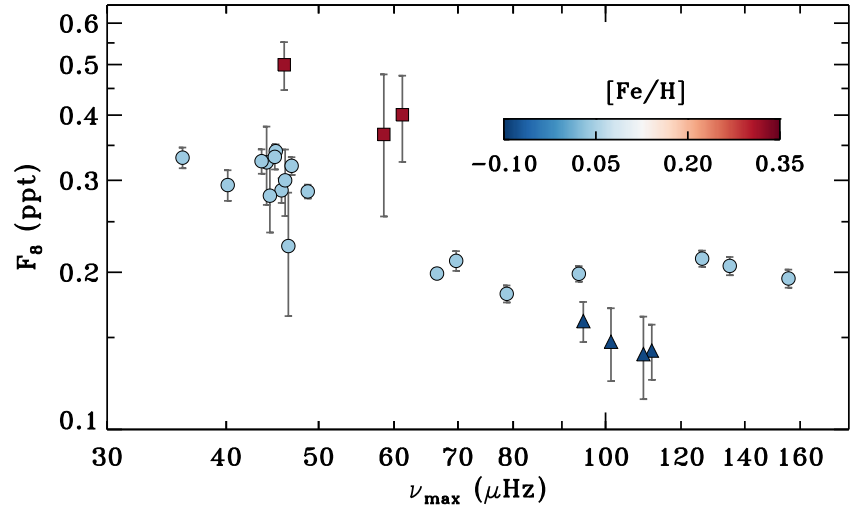

Fig. 9. Granulation flicker for timescales shorter than $8 \mathrm{~h}, F_{8}$, as a function of $v_{\max }$ for NGC 6791 (squares), NGC 6819 (circles) and NGC 6811 (triangles). The color-coding shows the cluster mean metallicity, similarly to Fig. 5 c. $1 \sigma$ measurement uncertainties on flicker are also shown.

of the total granulation signal on timescales shorter than $8 \mathrm{~h}$. The granulation flicker represents a measurement of the granulation activity that is independent of the background modeling adopted in Sect. 3.1. The result is shown in Fig. 9 as a function of $v_{\max }$ and $[\mathrm{Fe} / \mathrm{H}]$. To test the significance of the dependency on metallicity on top of those arising from a varying stellar mass and $v_{\max }$, we consider a scaling relation of the form

$F_{8}=\alpha\left(\frac{v_{\max }}{v_{\max , \odot}}\right)^{s}\left(\frac{M}{M_{\odot}}\right)^{t} \mathrm{e}^{u[\mathrm{Fe} / \mathrm{H}]}$,

with $\alpha$ a proportionality term in units of ppt, and $s, t, u$ exponents that need to be estimated. We thus apply the same Bayesian inference described in Sect. 5 to both the linearized models determined for $u=0$ (no metallicity effect) and $u \neq 0$ (metallicity included). The Bayesian model comparison between the two models considered, performed as described in Sect. 5.1, shows that the model including the metallicity term is significantly dominant over its competitor accounting only for $v_{\max }$ and stellar mass $\left(\ln O_{u \neq 0, u=0}=5.2\right)$. From our granulation flicker we find a metallicity exponent, $u=0.9 \pm 0.3$, which agrees, well within the $1 \sigma$ error, with that estimated for the meso-granulation amplitude from our detailed analysis of the background spectra presented in Sect. 6.1. This positive detection of the metallicity effect was possible despite the granulation flicker could be measured for only three of the high-metallicity stars of NGC 6791. We note that the uncertainties on $F_{8}$, and the errors on the parameters estimated from the fit to the whole sample, are about three times larger than for the analysis of the background spectra.

Lastly, we measure the granulation properties $a_{\text {meso }}$ and $b_{\text {meso }}$, together with $v_{\max }$, for an independent sample of 12 field RGs. These field stars have temperatures and metallicities available from the APOKASC catalog and all exhibit similar apparent magnitudes, which implies that the noise level in the stellar PSD is similar from star to star. This homogeneity in apparent magnitude, unlike our cluster RGs, is useful to isolate possible effects in estimating the background parameters that could arise from a different noise level in the data. The field stars are divided into two groups, the first one including six targets with solar metallicity (simulating the metallic content of the stars in NGC 6819 and NGC 6811), and the second group with six targets having super-solar metallicity, on average $\sim 0.24 \mathrm{dex}$, hence simulating a sample of stars with a metallic content close to that of NGC 6791. For the selected field stars we considered masses obtained from scaling using the asteroseismic parameters from 
the APOKASC DR13 catalog (Pinsonneault et al. 2014), and a $v_{\max }$ that is estimated through DIAMONDS using the same background model presented in Sect. 3.1. Each star of a set with similar metallicity has a relatively close comparative star in terms of mass and $v_{\max }$ in the other set of super-solar metallicity targets. Our choice of masses and $v_{\max }$ values for the field RGs allows us to soften the effect caused by a different stellar mass at constant surface gravity and to limit the tight dependence on $g / \sqrt{T_{\text {eff }}}$, which contributes each time that two targets having different metallicities are compared to one another. We observe a trend with metallicity for both meso-granulation amplitude and characteristic frequency similar to the one shown in this work. In particular, by considering the predictions obtained from the best meso-granulation amplitude scaling relation, Eq. (7), we obtain average residuals of $\sim 28 \mathrm{ppm}$ and $\sim 24 \mathrm{ppm}$ for the super-solar and solar metallicity samples, respectively. These estimates are similar to the dispersion of the residuals for the same scaling relation applied to the cluster RGs (about $28 \mathrm{ppm}$, see also Fig. C.1). The comparison done for the best mesogranulation frequency scaling relation, Eq. (10), shows that the average residuals are about $3.3 \mu \mathrm{Hz}$ and $3.5 \mu \mathrm{Hz}$ for the supersolar and the solar metallicity samples, respectively. Similarly to the case of the amplitudes, this result is again close to the dispersion of the residuals found in the cluster sample (about $2 \mu \mathrm{Hz}$, see Fig. C.2). We note that a dedicated and detailed analysis of the granulation activity for field stars that aims at calibrating the granulation scaling relations for a wide range of stellar parameters will be presented by Mathur et al. (in prep.). We therefore conclude that the systematic difference in amplitude and frequency of the meso-granulation signal that we observe in Figs. 5 and 6 could not be caused by either the different signalto-noise ratio in the PSDs of the cluster RGs or just the differing stellar masses.

\section{Discussion and conclusions}

From the results presented in Sect. 6 it appears clearly that the stellar metallicity has an important influence on the granulation activity in evolved cool stars (Fig. 8) and that meso-granulation and granulation properties depend solely on the conditions in the stellar atmospheres. We have shown that the cluster membership of the 60 RGs analyzed in this work is a powerful constraint that allows accurate calibration of the meso-granulation scaling relations in the metallicity range spanning from 0.8 times to about twice the solar metallicity ( $\sim 0.4 \mathrm{dex})$, and surface gravity $2.3 \leq \log g \leq 3.1$. This is because the stars in each cluster have the advantage of sharing rather homogeneous stellar properties of mass, temperature, and metallicity (see Appendix A and Table 1). The results of this work are therefore essential to identify and understand the underlying correlations among metallicity and stellar mass, surface gravity, and temperature. This study also sets the basis for the selection and detailed analysis of large samples of field stars spanning a wider range of fundamental stellar properties and evolutionary stages than the one covered here (Mathur et al., in prep.).

The signature of metallicity is statistically significant for both the amplitude and the characteristic frequency of the mesogranulation, hence of the granulation since the two components scale linearly across the entire $v_{\max }$ range investigated (see Sect. 3.1). From our sample, the effect of metallicity is observationally more enhanced for the amplitude parameter, for which we clearly observe a systematic difference between the closeto-solar metallicity stars and the super-solar metallicity ones (Figs. 5a, c). This is because metallicity has the opposite effect on the amplitudes than that of mass (Figs. 7 and 8, top panels), and because at the same time the high-metallicity cluster, NGC 6791, contains the stars with the lowest mass among the three clusters considered. In particular, $a_{\text {meso }}$ for stars belonging to NGC 6791 is about $60 \%$ larger than that of stars from NGC 6819 and NGC 6811, and this result is a combination of both metallicity and mass of the stars. These findings are also reflected in our measurements of the granulation flicker, $F_{8}$, where the high-metallicity stars show systematically larger flicker amplitudes than the low-metallicity ones (Fig. 9), and with a similar dependence on metallicity as estimated from the associated exponent $u$ of the scaling relations (see Sect. 6.3). Unlike for the amplitudes, mass and metallicity act in the same direction for the characteristic frequency $b_{\text {meso }}$, namely by decreasing the frequency scale of the meso-granulation, hence of the granulation, when they increase (Figs. 7 and 8, bottom panels). This also explains that the metallicity effect seen in Fig. 6a is less evident than that of the amplitudes, but it otherwise appears clear when the mass dependency is removed, as seen in Fig. $6 \mathrm{c}$ and in Fig. 8, bottom panel.

\subsection{Meso-granulation amplitude $a_{\text {meso }}$}

According to our analysis of the scaling relations presented in Sect. 4, a higher metallicity increases the granulation amplitude. The best model identified $\left(\mathcal{M}_{a, 2}\right.$, Eq. (7), see Table 2) gives a power law exponent for metallicity of $u=0.89$, with a precision of about $9 \%$. The positive value of the exponent is in qualitative agreement with theoretical modeling of realistic 3D stellar atmospheres by Collet et al. (2007). In particular, from the modeled granulation larger granules are found for higher metallicities due to the increased opacity. This implies that the amplitudes of the meso-granulation and granulation signals are higher because the associated disk-integrated brightness fluctuations scale as $n_{\text {gran }}^{-1 / 2}$, $n_{\text {gran }}$ being the number of granules observed on the stellar surface (Ludwig 2006). When the granulation scale is fixed, that is, when the atmospheric parameters are fixed, $n_{\text {gran }}$ scales with stellar radius as $R^{2}$ and the amplitude of the granulation signal therefore scales as $R^{-1}$, due to, for example, a change in stellar mass. The metallicity effect is also apparent in the new granulation analysis by Ludwig \& Steffen (2016), of 3D surface convection simulations of F-K stars, from the main sequence to the lower RGB and for solar and metal-poor, $[\mathrm{Fe} / \mathrm{H}]=-2$, compositions. They Fourier transformed time-series of specific bolometric intensity, integrated over the disk, and scaled to a star of solar radius to make it easy to apply their result to stars of any radius. For our sample of giants, the difference between bolometric and Kepler intensities will be rather small. From Ludwig \& Steffen (2016, Fig. 3), the increase in granulation amplitude with increasing metallicity is more pronounced toward larger values of surface gravity (or equivalently larger $v_{\max }$ ), which we also find in our observations (Figs. 5a, c, in the range 60-120 $\mu \mathrm{Hz}$ ).

We perform a direct comparison between our observed granulation amplitudes and the predictions from the 3D simulations by Ludwig \& Steffen (2016). We compare with the solar metallicity stars of our sample, from NGC 6819 and NGC 6811. For these stars we reduce the Kepler meso-granulation amplitudes to bolometric granulation amplitudes. We further reduce these amplitudes to that of a star with solar radius (having the same $T_{\text {eff }}$ and $\log g$ ) by multiplying by $\left(R_{*} / R_{\odot}\right)$. The stellar radii are computed according to Sharma et al. (2016; see also Appendix A). The bi-linear fits to the scaled bolometric amplitudes of Ludwig \& Steffen (2016), for both $[\mathrm{Fe} / \mathrm{H}]=0$ and -2 regimes 
are then applied to the $T_{\text {eff }}$ (from the SDSS-based temperature scale) and $\log g$ of all the targets of NGC 6819 and 6811 (see Tables A.2 and A.3) to obtain predicted estimates from the 3D simulations. The simulations from Ludwig \& Steffen (2016) exhibit 0.27 dex $(+85 \%)$ larger amplitudes for a 2.0 dex increase in metallicity in the range of temperature and surface gravities covered by our sample of RGs. By scaling the change in logarithmic amplitude down to the 0.32 dex increase in metallicity of our targets (from the two solar metallicity clusters, to NGC 6791) gives a $0.045 \mathrm{dex}(+11 \%)$ increase in amplitude. This agrees well with our observations, for which we find a $0.050 \mathrm{dex}(+12 \%)$ increase in amplitude between the clusters. Interestingly, our best amplitude model, $\mathcal{M}_{a, 2}$, is more sensitive to metallicity than $v_{\max }$ $(|s|<|u|$, see Table 2$)$.

As noted by K14, by exploiting the linear relation between $b_{\text {gran }}$ and $v_{\max }$, and by evaluating the background PSD at $v_{\max }$ from our Eq. (2), we can infer that $a_{\text {gran }} \propto v_{\max }^{-0.5}$. We can easily extend this relation to the meso-granulation amplitude since both $a_{\text {meso }}$ and $b_{\text {meso }}$ follow constant scalings from $a_{\text {gran }}$ and $b_{\text {gran }}$ (see, e.g., K14, and our discussions in Sects. 3.1 and 7.2). Our estimate of the exponent $s$ of the scaling relations for $a_{\text {meso }}$ is close to -0.5 (with -0.550 for $\mathcal{M}_{a, 1}$ and -0.593 for $\mathcal{M}_{a, 2}$ ), thus in better agreement with the expected dependency than what was found in previous works (see, e.g., K14).

According to our scaling relations we find that the effect of a varying mass at constant surface gravity on $a_{\text {meso }}$, could be well constrained from the population of cluster RGs thanks to the homogeneity of stellar masses found within each cluster (see Figs. 3, 5d, and 7, top panel). By taking into account RGs that exhibit, on average, a different mass depending on the cluster they belong to (see also Miglio et al. 2012; Corsaro et al. 2012 for more details), the effect of mass can be clearly disentangled from that of a different surface gravity and metallicity. The statistical error for the mass exponent, $t$, is about $19 \%$, comparable to that obtained by K14 using 100 times as many field stars but about one year shorter time-series. From the parameter estimation presented in Table 2, it appears that the impact of a varying mass on $a_{\text {meso }}$ is about a fourth in strength as compared to that of metallicity (for $\mathcal{M}_{a, 2}$ we have $4|t| \simeq|u|$ ). We note that since the amplitude of the signal scales with stellar radius as $R^{-1}$, for a constant surface gravity the amplitude scales as $M^{-1 / 2}$, which is not far from the estimates of the mass exponent $t$ for both models $\mathcal{M}_{a, 1}$ and $\mathcal{M}_{a, 2}(t=-0.67$ and -0.21 , respectively). Deviations from the expected exponent in mass are most likely due to temperature, surface gravity, and metallicity dependencies that are not entirely described by a $v_{\max }$ dependency.

Our analysis also has some implications on the connection between the granulation properties and the stellar evolutionary stage. On one hand, as visible from the residuals of the fits shown in Fig. C.1, we do not observe any systematic differences between RC and RGB stars. This confirms that the mesogranulation, hence also the granulation, are unaffected by the conditions in the stellar core because they are completely described by the atmospheric parameters and by the stellar radius, which produces an attenuation to the global power of granular fluctuations by a factor $R^{-2}$ due to the stochastic and incoherent nature of granulation (Trampedach et al. 2013). On the other hand, the coefficients $\beta$ estimated for the two meso-granulation amplitude models, and especially the best model $\mathcal{M}_{a, 2}$, suggest that the scaling relations might not be linear across the orders of magnitudes in surface gravity that separate the Sun from the evolved stars in our sample.

From our estimation of the background parameters shown in Fig. 5a, we notice two stars that have meso-granulation amplitudes significantly above than of similar stars, and significantly above our predictions from the scaling relations (by up to $25 \%$ as seen in Fig. C.1). These are the RGB star KIC 2437976 , with $v_{\max } \simeq 89 \mu \mathrm{Hz}$, and the RC star KIC 2437103, with $v_{\max } \simeq$ $29 \mu \mathrm{Hz}$, both in NGC 6791. Given the accuracy of the $v_{\max }$ and $\Delta v$ measurements for the stars, and their cluster membership, we discard errors in the mass computation as the source of the discrepancy. KIC 2437103 in particular, was originally misclassified from its position in the color-magnitude diagram as an RGB star, but then reclassified as RC from the properties of its oscillation (see Corsaro et al. 2012, for a detailed discussion). This star has the largest $a_{\text {meso }}$ of our sample, exceeding $700 \mathrm{ppm}$ (see Table B.1), which can in part be interpreted as a consequence of the combination of the low stellar mass, one of the smallest among all the stars that we analyzed (see also Fig. 5d), and the low $v_{\max }$. We conclude that the large amplitudes observed for these two stars could be the result of either a metallicity higher than the cluster mean metallicity adopted in our study, a possible blending with other sources (e.g., from a binary), or a combination of the two. Future abundance determinations, which are not yet available for the two mentioned stars, might help understanding the origin of the observed discrepancy.

\subsection{Meso-granulation characteristic frequency $b_{\text {meso }}$}

By analyzing four different scaling relations we have found that metallicity has a more dominant role than mass in determining the characteristic frequency of meso-granulation. Stellar mass has a rather weak effect, with an exponent $t=-0.38$, as compared to that of metallicity (from our best model $\mathcal{M}_{b, 4}$, Eq. (10), we have $|u| \simeq 3|t|)$, although still statistically significant (see Table 3). Once again, despite the relatively low number of stars in our sample as compared to that of K14, the metallicity exponent $u$ could be constrained to about $10 \%$, while the precision remains poorer on the mass exponent $t$ (about 16\%). In contrast to the meso-granulation amplitudes, we do not find a clear correspondence with the modeling performed by Ludwig \& Steffen (2016). This is because in the metallicity range explored by the authors $(-2 \leq[\mathrm{Fe} / \mathrm{H}] \leq 0)$ the granulation characteristic frequency appears to have an almost negligible dependency upon metallicity, with variations of $\sim 6 \%$, comparable to the level of the residuals found from the scaling relations investigated in this work. Interestingly, for our best model we find that $|u| \simeq 1.3|s|$, indicating that $v_{\max }$ and $[\mathrm{Fe} / \mathrm{H}]$ are almost equally important in determining $b_{\text {meso }}$. We conclude that in order to assess our results on the characteristic frequency of the granulation activity, more dedicated theoretical investigations would be required, for example with 3D simulations covering the atmospheric parameters of our sample of cluster stars, and by extracting granulation parameters from the Fourier spectra of time-series obtained from such simulations, based on the background fitting models used in Sect. 3.1. Mathur et al. (in prep.) is investigating the metallicity effect, based on Kepler observations of field stars, and the convection simulations by Trampedach et al. (2013; see Mathur et al. 2011, for the granulation parameters for this grid) to cover a wider range of metallicity, surface gravities, and temperatures, than that of Ludwig \& Steffen (2016).

The exponent $s$, of $v_{\max }$, is 0.898 for our best model $\mathcal{M}_{b, 4}$ (incorporating both metallicity and mass in the fit) and shows that the relation between $b_{\text {meso }}$ and $v_{\max }$ is nearly linear as a first approximation, thus validating the presence of a tight connection between the granulation characteristic frequency and the surface gravity of the star. As argued by Kjeldsen \& Bedding (2011) and by Mathur et al. (2011, see also K14), this confirms 
that convection cells travel for a vertical distance that is proportional to the pressure scale height, $H_{\mathrm{p}}$, at a speed proportional to the speed of sound $c_{\mathrm{s}}$ to first approximation. For explaining this result, we consider that the derived characteristic frequency of the granulation can be expressed as $b_{\text {gran }} \propto c_{\mathrm{s}} / H_{\mathrm{p}}$. Taking into account the seismic scaling relation $v_{\max } \propto g / \sqrt{T_{\text {eff }}}$ (Brown et al. 1991), and the relations $c_{\mathrm{s}} \propto \sqrt{T_{\text {eff }}}$ and $H_{\mathrm{p}} \propto T_{\text {eff }} / g$, we thus have that $b_{\text {gran }} \propto v_{\max }$ because it is the same convection that excites the acoustic oscillations and gives rise to the granulation at the surface. This in turn implies that $b_{\text {meso }} \propto v_{\max }$, because the meso-granulation represents conglomerations of a certain number of granules, hence meso-granulation and granulation have time scales that are proportional to each other, $b_{\text {meso }} \propto b_{\text {gran }}$, as we also quantify in Sect. 3.1.

Like $a_{\text {meso }}$, the distribution of $b_{\text {meso }}$ for RGB and RC stars is similar (e.g., see Fig. C.2). Once again this indicates that granulation and meso-granulation inherently depend on the atmospheric conditions only, with no effect from the different core structures between RGB and RC stages of stellar evolution. In addition, the power of the granulation signal decreases with stellar radius due to the averaging over an increasing number of incoherent granules. This manifests in our fits from the best model $\mathcal{M}_{\mathrm{b}, 4}$, as a negative mass-exponent $t$.

Acknowledgements. E.C. is funded by the European Union's Horizon 2020 research and innovation program under the Marie Sklodowska-Curie grant agreement No. 664931 and by the European Community's Seventh Framework Programme (FP7/2007-2013) under grant agreement No. 312844 (SPACEINN). S.M. acknowledges support from NASA grants NNX12AE17G and NNX15AF13G and NSF grant AST-1411685. R.A.G. received funding from the CNES GOLF and PLATO grants at CEA and from the ANR (Agence Nationale de la Recherche, France) program IDEE (No. ANR-12-BS05-0008) "Interaction Des Étoiles et des Exoplanètes". Funding for the Sloan Digital Sky Survey IV has been provided by the Alfred P. Sloan Foundation, the US Department of Energy Office of Science, and the Participating Institutions. SDSS acknowledges support and resources from the Center for High-Performance Computing at the University of Utah. The SDSS web site is www.sdss.org. SDSS is managed by the Astrophysical Research Consortium for the Participating Institutions of the SDSS Collaboration including the Brazilian Participation Group, the Carnegie Institution for Science, Carnegie Mellon Univer sity, the Chilean Participation Group, the French Participation Group, HarvardSmithsonian Center for Astrophysics, Instituto de Astrofísica de Canarias, The Johns Hopkins University, Kavli Institute for the Physics and Mathematics of the Universe (IPMU)/University of Tokyo, Lawrence Berkeley National Laboratory, Leibniz Institut für Astrophysik Potsdam (AIP), Max-Planck-Institut für Astronomie (MPIA Heidelberg), Max-Planck-Institut für Astrophysik (MPA Garching), Max-Planck-Institut für Extraterrestrische Physik (MPE), National Astronomical Observatories of China, New Mexico State University, New York University, University of Notre Dame, Observatório Nacional/MCTI, The Ohio State University, Pennsylvania State University, Shanghai Astronomical Observatory, United Kingdom Participation Group, Universidad Nacional Autónoma de México, University of Arizona, University of Colorado Boulder, University of Oxford, University of Portsmouth, University of Utah, University of Virginia University of Washington, University of Wisconsin, Vanderbilt University, and Yale University.

\section{References}

Aigrain, S., Favata, F., \& Gilmore, G. 2004, A\&A, 414, 1139

Baglin, A., Michel, E., Auvergne, M., \& COROT Team. 2006, in Proceedings of SOHO 18/GONG 2006/HELAS I, Beyond the spherical Sun, ESA SP, 624, 34

Basri, G., Walkowicz, L. M., Batalha, N., et al. 2010, ApJ, 713, L155 Bastien, F. A., Stassun, K. G., Basri, G., \& Pepper, J. 2013, Nature, 500, 427 Bastien, F. A., Stassun, K. G., Basri, G., \& Pepper, J. 2016, ApJ, 818, 43 Basu, S., Grundahl, F., Stello, D., et al. 2011, ApJ, 729, L10 Bedding, T. R., Mosser, B., Huber, D., et al. 2011, Nature, 471, 608 Belkacem, K., Goupil, M. J., Dupret, M. A., et al. 2011, A\&A, 530, A142 Blanton, M. R., Bershady, M. A., Abolfathi, B., et al. 2017, AJ, 154, 28 Bonanno, A., Corsaro, E., \& Karoff, C. 2014, A\&A, 571, A35

Borucki, W. J., Koch, D., Basri, G., et al. 2010, Science, 327, 977
Bragaglia, A., Carretta, E., Gratton, R. G., et al. 2001, AJ, 121, 327 Brogaard, K., Bruntt, H., Grundahl, F., et al. 2011, A\&A, 525, A2 Brogaard, K., VandenBerg, D. A., Bruntt, H., et al. 2012, A\&A, 543, A106 Brown, T. M., Gilliland, R. L., Noyes, R. W., \& Ramsey, L. W. 1991, ApJ, 368, 599

Brown, T. M., Latham, D. W., Everett, M. E., \& Esquerdo, G. A. 2011, AJ, 142, 112

Collet, R., Asplund, M., \& Trampedach, R. 2007, A\&A, 469, 687

Corsaro, E., \& De Ridder, J. 2014, A\&A, 571, A71

Corsaro, E., Stello, D., Huber, D., et al. 2012, ApJ, 757, 190

Corsaro, E., Fröhlich, H.-E., Bonanno, A., et al. 2013, MNRAS, 430, 2313

Corsaro, E., De Ridder, J., \& García, R. A. 2015, A\&A, 579, A83

Corsaro, E., Lee, Y.-N., García, R. A., et al. 2017, Nat. Astron., 1, 0064

García, R. A., Hekker, S., Stello, D., et al. 2011, MNRAS, 414, L6

García, R. A., Mathur, S., Pires, S., et al. 2014, A\&A, 568, A10

García Pérez, A. E., Allende Prieto, C., Holtzman, J. A., et al. 2016, AJ, 151, 144

Gaulme, P., Appourchaux, T., \& Boumier, P. 2009, A\&A, 506, 7

Gaulme, P., McKeever, J., Jackiewicz, J., et al. 2016, ApJ, 832, 121

Guggenberger, E., Hekker, S., Basu, S., \& Bellinger, E. 2016, MNRAS, 460, 4277

Gunn, J. E., Siegmund, W. A., Mannery, E. J., et al. 2006, AJ, 131, 2332

Harvey, J. 1985, in Future Missions in Solar, Heliospheric \& Space Plasma Physics, eds. E. Rolfe, \& B. Battrick, ESA SP, 235, 199

Hekker, S., Basu, S., Stello, D., et al. 2011, A\&A, 530, A100

Hekker, S., Elsworth, Y., Mosser, B., et al. 2012, A\&A, 544, A90

Herschel, W. 1801, Philosophical Transactions of the Royal Society of London Series I, 91, 265

Holtzman, J. A., Shetrone, M., Johnson, J. A., et al. 2015, AJ, 150, 148

Huber, D., Bedding, T. R., Stello, D., et al. 2011, ApJ, 743, 143

Jenkins, J. M., Caldwell, D. A., Chandrasekaran, H., et al. 2010, ApJ, 713, L120

Kallinger, T., \& Matthews, J. M. 2010, ApJ, 711, L35

Kallinger, T., De Ridder, J., Hekker, S., et al. 2014, A\&A, 570, A41 (K14)

Kallinger, T., Hekker, S., Garcia, R. A., Huber, D., \& Matthews, J. M. 2016, Sci. Adv., 2, 1500654

Karoff, C., Campante, T. L., Ballot, J., et al. 2013, ApJ, 767, 34

Kjeldsen, H., \& Bedding, T. R. 2011, A\&A, 529, L8

Ludwig, H.-G. 2006, A\&A, 445, 661

Ludwig, H.-G., \& Steffen, M. 2016, Astron. Nachr., 337, 844

Magic, Z., Chiavassa, A., Collet, R., \& Asplund, M. 2015a, A\&A, 573, A90

Magic, Z., Weiss, A., \& Asplund, M. 2015b, A\&A, 573, A89

Majewski, S., et al. 2017, AJ, submitted

Mathur, S., García, R. A., Catala, C., et al. 2010, A\&A, 518, A53

Mathur, S., Hekker, S., Trampedach, R., et al. 2011, ApJ, 741, 119

Mathur, S., Huber, D., Batalha, N. M., et al. 2017, ApJS, 229, 30

Miglio, A., Brogaard, K., Stello, D., et al. 2012, MNRAS, 419, 2077

Milliman, K. E., Mathieu, R. D., Geller, A. M., et al. 2014, AJ, 148, 38

Molenda-Żakowicz, J., Brogaard, K., Niemczura, E., et al. 2014, MNRAS, 445 , 2446

Mosser, B., Michel, E., Belkacem, K., et al. 2013, A\&A, 550, A126

Mosser, B., Benomar, O., Belkacem, K., et al. 2014, A\&A, 572, L5

Nidever, D. L., Holtzman, J. A., Allende Prieto, C., et al. 2015, AJ, 150, 173

Perryman, M. A. C., de Boer, K. S., Gilmore, G., et al. 2001, A\&A, 369, 339

Pinsonneault, M. H., An, D., Molenda-Żakowicz, J., et al. 2012, ApJS, 199, 30

Pinsonneault, M. H., Elsworth, Y., Epstein, C., et al. 2014, ApJS, 215, 19

Pires, S., Mathur, S., García, R. A., et al. 2015, A\&A, 574, A18

Samadi, R., Belkacem, K, \& Ludwig, H.-G. 2013a, A\&A, 559, A39

Samadi, R., Belkacem, K., Ludwig, H.-G., et al. 2013b, A\&A, 559, A40

Scott, J. G., \& Berger, J. O. 2010, Ann. Stat., 38, 2587

SDSS Collaboration (Albareti, F. D., et al.) 2016, ApJS, submitted [arXiv: 1608.02013]

Sharma, S., Stello, D., Bland-Hawthorn, J., Huber, D., \& Bedding, T. R. 2016 , ApJ, 822, 15

Stello, D., Meibom, S., Gilliland, R. L., et al. 2011, ApJ, 739, 13

Tanner, J. D., Basu, S., \& Demarque, P. 2013, ApJ, 778, 117

Tayar, J., Somers, G., Pinsonneault, M. H., et al. 2017, ApJ, 840, 17

Trampedach, R., Christensen-Dalsgaard, J., Nordlund, A., \& Stein, R. F. 1998 , in The First MONS Workshop: Science with a Small Space Telescope, eds. H. Kjeldsen, \& T. R. Bedding, 59

Trampedach, R., Asplund, M., Collet, R., Nordlund, Å., \& Stein, R. F. 2013, ApJ, 769, 18

Trampedach, R., Stein, R. F., Christensen-Dalsgaard, J., Nordlund, Å., \& Asplund, M. 2014, MNRAS, 442, 805

Ulrich, R. K. 1986, ApJ, 306, L37

Vrard, M., Mosser, B., \& Samadi, R. 2016, A\&A, 588, A87

White, T. R., Bedding, T. R., Stello, D., et al. 2011, ApJ, 743, 161

Zasowski, G., Johnson, J. A., Frinchaboy, P. M., et al. 2013, AJ, 146, 81 
E. Corsaro et al.: Metallicity effect on stellar granulation from cluster red giants

\section{Appendix A: Stellar atmospheric parameters and masses}

Our selection of stellar atmospheric parameters from the different sources presented in Sect. 2 is shown in Tables A.1-A.3, for NGC 6791, NGC 6819, and NGC 6811, respectively. The values for surface gravity, $\log g$, are also provided for completeness and are derived from corrected stellar masses and radii computed according to Sharma et al. (2016). The $1 \sigma$ uncertainty in $\log g$ is determined from a standard propagation from those of corrected masses and radii.

Table A.1. Atmospheric parameters $T_{\text {eff }}$ (from SDSS-based temperature scale, see Sect. 2.2, with a total uncertainty of $69 \mathrm{~K}$ for all stars that are not marked by an apex), [Fe/H] from ASPCAP where available, and corrected stellar masses for NGC 6791 following Sharma et al. (2016).

\begin{tabular}{|c|c|c|c|c|c|}
\hline KIC ID & $\begin{array}{l}T_{\text {eff }} \\
(\mathrm{K})\end{array}$ & {$[\mathrm{Fe} / \mathrm{H}]$} & $\begin{array}{l}\text { Mass } \\
\left(M_{\odot}\right)\end{array}$ & $\begin{array}{l}\log g \\
(\operatorname{dex})\end{array}$ & State \\
\hline $2297384^{a}$ & 4504 & $0.38 \pm 0.02$ & $1.06 \pm 0.04$ & $2.37 \pm 0.02$ & $\mathrm{RC}$ \\
\hline $2297825^{a}$ & 4479 & $0.31 \pm 0.02$ & $0.95 \pm 0.05$ & $2.37 \pm 0.02$ & $\mathrm{RC}$ \\
\hline 2435987 & 4427 & $0.28 \pm 0.02$ & $1.09 \pm 0.03$ & $2.46 \pm 0.01$ & RGB \\
\hline 2436097 & 4402 & - & $1.11 \pm 0.03$ & $2.51 \pm 0.02$ & RGB \\
\hline 2436417 & 4460 & $0.31 \pm 0.02$ & $1.04 \pm 0.04$ & $2.32 \pm 0.02$ & $\mathrm{RC}$ \\
\hline 2436458 & 4350 & - & $1.05 \pm 0.03$ & $2.45 \pm 0.01$ & RGB \\
\hline 2436676 & 4573 & - & $0.97 \pm 0.03$ & $3.01 \pm 0.01$ & RGB \\
\hline 2436732 & 4503 & - & $1.10 \pm 0.03$ & $2.37 \pm 0.02$ & $\mathrm{RC}$ \\
\hline 2436818 & 4610 & - & $1.04 \pm 0.03$ & $2.87 \pm 0.01$ & RGB \\
\hline 2437103 & 4503 & - & $0.83 \pm 0.03$ & $2.35 \pm 0.02$ & $\mathrm{RC}$ \\
\hline 2437240 & 4459 & - & $1.15 \pm 0.03$ & $2.55 \pm 0.01$ & RGB \\
\hline $2437270^{a}$ & 4499 & - & $1.26 \pm 0.05$ & $2.73 \pm 0.02$ & RGB \\
\hline 2437325 & 4484 & - & $1.04 \pm 0.03$ & $2.86 \pm 0.01$ & RGB \\
\hline 2437353 & 4520 & $0.30 \pm 0.02$ & $1.10 \pm 0.03$ & $2.39 \pm 0.01$ & $\mathrm{RC}$ \\
\hline 2437564 & 4467 & $0.32 \pm 0.02$ & $1.12 \pm 0.03$ & $2.39 \pm 0.02$ & $\mathrm{RC}$ \\
\hline $2437589^{a}$ & 4508 & - & $1.44 \pm 0.06$ & $2.55 \pm 0.02$ & RGB \\
\hline 2437804 & 4439 & $0.35 \pm 0.03$ & $1.02 \pm 0.03$ & $2.31 \pm 0.02$ & $\mathrm{RC}$ \\
\hline 2437933 & 4534 & - & $1.11 \pm 0.03$ & $2.92 \pm 0.01$ & RGB \\
\hline 2437957 & 4556 & - & $1.03 \pm 0.03$ & $2.86 \pm 0.01$ & RGB \\
\hline $2437972^{a}$ & 4543 & - & $1.09 \pm 0.04$ & $2.82 \pm 0.02$ & RGB \\
\hline 2437976 & 4478 & - & $1.05 \pm 0.03$ & $2.84 \pm 0.01$ & RGB \\
\hline 2437987 & 4517 & - & $1.13 \pm 0.05$ & $2.38 \pm 0.02$ & $\mathrm{RC}$ \\
\hline $2438038^{a}$ & 4450 & - & $1.09 \pm 0.04$ & $2.68 \pm 0.02$ & RGB \\
\hline 2438051 & 4524 & $0.30 \pm 0.02$ & $1.14 \pm 0.07$ & $2.37 \pm 0.03$ & $\mathrm{RC}$ \\
\hline 2438333 & 4473 & $0.32 \pm 0.02$ & $1.08 \pm 0.03$ & $2.67 \pm 0.01$ & RGB \\
\hline 2569055 & 4479 & $0.32 \pm 0.02$ & $1.09 \pm 0.04$ & $2.38 \pm 0.02$ & $\mathrm{RC}$ \\
\hline 2569945 & 4507 & $0.33 \pm 0.02$ & $0.97 \pm 0.04$ & $2.37 \pm 0.02$ & $\mathrm{RC}$ \\
\hline $2570094^{a}$ & 4485 & - & $1.16 \pm 0.04$ & $2.72 \pm 0.02$ & RGB \\
\hline 2570244 & 4515 & - & $1.09 \pm 0.03$ & $2.91 \pm 0.01$ & RGB \\
\hline 2570384 & 4519 & $0.32 \pm 0.02$ & $0.81 \pm 0.05$ & $2.65 \pm 0.03$ & RGB \\
\hline
\end{tabular}

Notes. Surface gravities, $\log g$, and corresponding $1 \sigma$ uncertainties, are also provided using stellar radii computed according to Sharma et al. (2016). The evolutionary state specifies whether a star is RC or RGB, as classified by Corsaro et al. $(2012,2017) .{ }^{(a)}$ The source for temperature is the $(V-K)$ color (Hekker et al. 2011), with an adopted uncertainty of 110 K. See Sect. 2.2 for more details.
Table A.2. Same as in Table A.1 but for NGC 6819.

\begin{tabular}{cccccl}
\hline \hline KIC ID & $\begin{array}{c}T_{\text {eff }} \\
(\mathrm{K})\end{array}$ & {$[\mathrm{Fe} / \mathrm{H}]$} & $\begin{array}{c}\text { Mass } \\
\left(M_{\odot}\right)\end{array}$ & $\begin{array}{c}\log g \\
(\mathrm{dex})\end{array}$ & State \\
\hline 4937056 & 4844 & $0.01 \pm 0.02$ & $1.56 \pm 0.07$ & $2.57 \pm 0.02$ & $\mathrm{RC}$ \\
4937770 & 5033 & $-0.02 \pm 0.02$ & $1.93 \pm 0.08$ & $2.88 \pm 0.02$ & $\mathrm{RC}$ \\
5023953 & 4834 & $0.07 \pm 0.02$ & $1.84 \pm 0.05$ & $2.59 \pm 0.01$ & $\mathrm{RC}$ \\
5024327 & 4865 & - & $1.50 \pm 0.05$ & $2.55 \pm 0.02$ & $\mathrm{RC}$ \\
5024404 & 4798 & $0.07 \pm 0.02$ & $1.52 \pm 0.04$ & $2.57 \pm 0.01$ & $\mathrm{RC}$ \\
5024414 & 5031 & - & $2.40 \pm 0.05$ & $2.81 \pm 0.01$ & $\mathrm{RC}$ \\
5024476 & 4968 & $0.05 \pm 0.02$ & $2.21 \pm 0.05$ & $2.73 \pm 0.01$ & $\mathrm{RC}$ \\
5024582 & 4873 & - & $1.54 \pm 0.04$ & $2.57 \pm 0.01$ & $\mathrm{RC}$ \\
5024967 & 4797 & - & $1.52 \pm 0.05$ & $2.56 \pm 0.02$ & $\mathrm{RC}$ \\
5111718 & 4916 & $0.08 \pm 0.02$ & $1.60 \pm 0.04$ & $3.04 \pm 0.01$ & $\mathrm{RGB}$ \\
5111949 & 4804 & $0.07 \pm 0.03$ & $1.49 \pm 0.04$ & $2.57 \pm 0.01$ & $\mathrm{RC}$ \\
5112072 & 4937 & $0.01 \pm 0.03$ & $1.60 \pm 0.03$ & $3.01 \pm 0.01$ & $\mathrm{RGB}$ \\
5112361 & 4924 & $-0.03 \pm 0.03$ & $1.87 \pm 0.04$ & $2.75 \pm 0.01$ & $\mathrm{RGB}$ \\
5112373 & 4826 & $0.04 \pm 0.03$ & $1.51 \pm 0.04$ & $2.55 \pm 0.01$ & $\mathrm{RC}$ \\
5112387 & 4808 & $0.05 \pm 0.02$ & $1.49 \pm 0.04$ & $2.55 \pm 0.01$ & $\mathrm{RC}$ \\
5112401 & 4797 & $0.01 \pm 0.03$ & $1.32 \pm 0.04$ & $2.46 \pm 0.02$ & $\mathrm{RC}$ \\
5112467 & 4841 & $0.05 \pm 0.03$ & $1.51 \pm 0.04$ & $2.57 \pm 0.01$ & $\mathrm{RC}$ \\
5112491 & 4894 & $0.00 \pm 0.02$ & $1.50 \pm 0.04$ & $2.55 \pm 0.01$ & $\mathrm{RC}$ \\
5112730 & 4794 & $0.06 \pm 0.02$ & $1.54 \pm 0.04$ & $2.54 \pm 0.01$ & $\mathrm{RC}$ \\
5112938 & 4798 & $0.06 \pm 0.02$ & $1.41 \pm 0.04$ & $2.55 \pm 0.01$ & $\mathrm{RC}$ \\
5112950 & 4746 & $0.07 \pm 0.02$ & $1.51 \pm 0.04$ & $2.51 \pm 0.02$ & $\mathrm{RC}$ \\
5112974 & 4790 & $0.03 \pm 0.02$ & $1.50 \pm 0.04$ & $2.50 \pm 0.01$ & $\mathrm{RC}$ \\
5113441 & 4829 & $0.08 \pm 0.02$ & $1.55 \pm 0.03$ & $3.09 \pm 0.01$ & $\mathrm{RGB}$ \\
5200152 & 4927 & $0.05 \pm 0.02$ & $1.51 \pm 0.04$ & $2.56 \pm 0.02$ & $\mathrm{RC}$ \\
\hline & & & & & \\
\hline
\end{tabular}

Table A.3. Same as in Table A.1 but for NGC 6811.

\begin{tabular}{cccccc}
\hline \hline KIC ID & $\begin{array}{c}T_{\text {eff }} \\
(\mathrm{K})\end{array}$ & {$[\mathrm{Fe} / \mathrm{H}]$} & $\begin{array}{c}\text { Mass } \\
\left(M_{\odot}\right)\end{array}$ & $\begin{array}{c}\log g \\
(\mathrm{dex})\end{array}$ & State \\
\hline 9532903 & 5106 & $-0.06 \pm 0.02$ & $2.27 \pm 0.06$ & $2.89 \pm 0.01$ & $\mathrm{RC}$ \\
9534041 & 5144 & $-0.11 \pm 0.02$ & $2.72 \pm 0.06$ & $2.96 \pm 0.01$ & $\mathrm{RC}$ \\
9655101 & 5067 & $-0.11 \pm 0.03$ & $2.54 \pm 0.06$ & $2.92 \pm 0.01$ & $\mathrm{RC}$ \\
9716090 & 5084 & - & $2.25 \pm 0.05$ & $2.95 \pm 0.01$ & $\mathrm{RC}$ \\
9716522 & 4985 & $-0.06 \pm 0.03$ & $2.47 \pm 0.06$ & $2.64 \pm 0.01$ & $\mathrm{RC}$ \\
9776739 & 5152 & - & $2.27 \pm 0.06$ & $2.89 \pm 0.01$ & $\mathrm{RC}$ \\
\hline
\end{tabular}

Notes. Sources for temperature and metallicities are as in Table A.1. The evolutionary state of the stars KIC 9776739 and KIC 9716090 is provided by Molenda-Żakowicz et al. (2014) and confirmed by Corsaro et al. (in prep.). 


\section{Appendix B: Results for the background fitting}

The background parameters $a_{\text {meso }}, b_{\text {meso }}, v_{\text {max }}$ derived by means of DIAMONDS for the entire sample of 60 cluster RGs analyzed in this work are listed for NGC 6791 in Table B.1, NGC 6819 in Table B.2, and NGC 6811 in Table B.3.

Following the definitions presented by Corsaro \& De Ridder (2014), the configuring parameters used in DIAMONDS are: initial enlargement fraction $f_{0}=1.3$, shrinking rate $\alpha=0.02$, number of live points $N_{\text {live }}=500$, number of clusters $1 \leq N_{\text {clust }} \leq 10$, number of total drawing attempts $M_{\text {attempts }}=10^{4}$, number of nested iterations before the first clustering $M_{\text {init }}=1500$, and number of nested iterations with the same clustering $M_{\text {same }}=50$.

Table B.1. Median values with corresponding $68.3 \%$ Bayesian credible intervals of the background parameters $a_{\text {gran }}, b_{\text {gran }}, v_{\max }$ for the RGs of the open cluster NGC 6791, as derived using DIAMONDS.

\begin{tabular}{|c|c|c|c|}
\hline KIC ID & $\begin{array}{l}a_{\text {meso }} \\
(\mathrm{ppm})\end{array}$ & $\begin{array}{l}b_{\text {meso }} \\
(\mu \mathrm{Hz})\end{array}$ & $\begin{array}{c}v_{\max } \\
(\mu \mathrm{Hz})\end{array}$ \\
\hline 2297384 & $561.9_{-16.5}^{+17.4}$ & $10.5_{-0.4}^{+0.5}$ & $30.6_{-0.2}^{+0.2}$ \\
\hline 2297825 & $583.8_{-18.3}^{+19.0}$ & $10.4_{-0.5}^{+0.5}$ & $30.4_{-0.3}^{+0.3}$ \\
\hline 2435987 & $477.8_{-9.9}^{+9.9}$ & $10.4_{-0.4}^{+0.4}$ & $37.8_{-0.2}^{+0.2}$ \\
\hline 2436097 & $465.7_{-12.0}^{+11.1}$ & $12.4_{-0.6}^{+0.6}$ & $42.1_{-0.2}^{+0.3}$ \\
\hline 2436417 & $604.0_{-25.6}^{+28.5}$ & $10.1_{-0.7}^{+0.7}$ & $27.4_{-0.2}^{+0.2}$ \\
\hline 2436458 & $499.2_{-10.3}^{+10.2}$ & $10.8_{-0.4}^{+0.4}$ & $37.1_{-0.2}^{+0.2}$ \\
\hline 2436676 & $223.8_{-6.9}^{+6.8}$ & $35.8_{-1.0}^{+1.0}$ & $131.5_{-0.7}^{+0.6}$ \\
\hline 2436732 & $580.6_{-10.9}^{+12.6}$ & $8.9_{-0.3}^{+0.3}$ & $30.3_{-0.2}^{+0.2}$ \\
\hline 2436818 & $276.2_{-4.8}^{+5.1}$ & $26.4_{-0.5}^{+0.6}$ & $95.5_{-0.5}^{+0.5}$ \\
\hline 2437103 & $711.3_{-21.5}^{+20.8}$ & $8.1_{-0.4}^{+0.5}$ & $28.8_{-0.2}^{+0.2}$ \\
\hline 2437240 & $448.3_{-9.7}^{+9.1}$ & $12.9_{-0.5}^{+0.4}$ & $46.0_{-0.2}^{+0.2}$ \\
\hline 2437270 & $311.5_{-5.0}^{+6.3}$ & $16.2_{-0.5}^{+0.5}$ & $69.9_{-0.2}^{+0.2}$ \\
\hline 2437325 & $276.9_{-6.4}^{+5.8}$ & $23.3_{-0.4}^{+0.4}$ & $94.1_{-0.2}^{+0.2}$ \\
\hline 2437353 & $508.9_{-11.8}^{+10.3}$ & $9.1_{-0.3}^{+0.4}$ & $31.7_{-0.2}^{+0.2}$ \\
\hline 2437564 & $537.8_{-17.3}^{+15.4}$ & $9.9_{-0.5}^{+0.5}$ & $32.0_{-0.2}^{+0.2}$ \\
\hline 2437589 & $364.0_{-8.2}^{+7.5}$ & $14.5_{-0.5}^{+0.5}$ & $46.1_{-0.3}^{+0.3}$ \\
\hline 2437804 & $612.6_{-14.9}^{+16.3}$ & $8.1_{-0.3}^{+0.4}$ & $26.7_{-0.2}^{+0.2}$ \\
\hline 2437933 & $262.4_{-5.9}^{+6.8}$ & $28.9_{-0.9}^{+1.0}$ & $108.4_{-0.3}^{+0.3}$ \\
\hline 2437957 & $279.2_{-14.6}^{+22.5}$ & $28.4_{-1.1}^{+1.2}$ & $92.7_{-0.4}^{+0.3}$ \\
\hline 2437972 & $358.8_{-21.5}^{+28.4}$ & $19.8_{-1.0}^{+0.9}$ & $85.2_{-0.3}^{+0.3}$ \\
\hline 2437976 & $412.7_{-22.3}^{+24.8}$ & $26.2_{-1.5}^{+1.7}$ & $89.3_{-0.3}^{+0.4}$ \\
\hline 2437987 & $576.5_{-19.6}^{+18.7}$ & $8.5_{-0.6}^{+0.5}$ & $31.0_{-0.4}^{+0.4}$ \\
\hline 2438038 & $351.7_{-7.1}^{+6.8}$ & $15.3_{-0.5}^{+0.5}$ & $62.5_{-0.2}^{+0.2}$ \\
\hline 2438051 & $590.3_{-18.2}^{+15.8}$ & $9.3_{-0.4}^{+0.4}$ & $30.1_{-0.5}^{+0.6}$ \\
\hline 2438333 & $372.1_{-6.2}^{+7.0}$ & $15.1_{-0.4}^{+0.4}$ & $61.2_{-0.2}^{+0.2}$ \\
\hline 2569055 & $564.8_{-16.5}^{+16.9}$ & $9.5_{-0.5}^{+0.5}$ & $31.0_{-0.3}^{+0.3}$ \\
\hline 2569945 & $551.0_{-18.9}^{+19.4}$ & $10.3_{-0.5}^{+0.6}$ & $30.4_{-0.4}^{+0.4}$ \\
\hline 2570094 & $324.2_{-7.8}^{+7.6}$ & $16.0_{-0.5}^{+0.5}$ & $68.1_{-0.2}^{+0.2}$ \\
\hline 2570244 & $245.2_{-5.8}^{+5.9}$ & $24.0_{-1.0}^{+0.7}$ & $105.8_{-0.4}^{+0.4}$ \\
\hline 2570384 & $410.9_{-19.7}^{+28.4}$ & $19.1_{-1.4}^{+1.7}$ & $58.5_{-1.1}^{+0.9}$ \\
\hline
\end{tabular}

Table B.2. Same as in Table B.1 but for NGC 6819.

\begin{tabular}{|c|c|c|c|}
\hline KIC ID & $\begin{array}{l}a_{\text {meso }} \\
(\mathrm{ppm})\end{array}$ & $\begin{array}{l}b_{\text {meso }} \\
(\mu \mathrm{Hz})\end{array}$ & $\begin{array}{c}v_{\max } \\
(\mu \mathrm{Hz})\end{array}$ \\
\hline 4937056 & $344.8_{-7.4}^{+7.8}$ & $14.1_{-0.6}^{+0.6}$ & $46.3_{-0.6}^{+0.7}$ \\
\hline 4937770 & $162.9_{-8.7}^{+10.2}$ & $32.9_{-2.9}^{+2.1}$ & $93.8_{-1.0}^{+1.1}$ \\
\hline 5023953 & $301.9_{-3.7}^{+3.9}$ & $14.1_{-0.3}^{+0.3}$ & $48.7_{-0.2}^{+0.2}$ \\
\hline 5024327 & $344.0_{-8.4}^{+8.4}$ & $15.5_{-0.5}^{+0.6}$ & $44.4_{-0.3}^{+0.4}$ \\
\hline 5024404 & $325.7_{-4.7}^{+4.6}$ & $12.6_{-0.3}^{+0.3}$ & $47.0_{-0.2}^{+0.2}$ \\
\hline 5024414 & $180.4_{-3.2}^{+3.2}$ & $27.5_{-1.5}^{+1.6}$ & $78.8_{-0.2}^{+0.2}$ \\
\hline 5024476 & $189.0_{-4.0}^{+4.2}$ & $20.5_{-0.4}^{+0.4}$ & $66.6_{-0.3}^{+0.3}$ \\
\hline 5024582 & $311.2_{-4.1}^{+4.2}$ & $13.6_{-0.3}^{+0.3}$ & $46.5_{-0.2}^{+0.2}$ \\
\hline 5024967 & $320.5_{-7.1}^{+6.3}$ & $14.7_{-0.6}^{+0.5}$ & $45.7_{-0.4}^{+0.4}$ \\
\hline 5111718 & $172.2_{-1.8}^{+1.9}$ & $36.5_{-0.8}^{+0.6}$ & $135.0_{-0.3}^{+0.3}$ \\
\hline 5111949 & $351.3_{-5.6}^{+5.0}$ & $14.6_{-0.4}^{+0.4}$ & $46.8_{-0.2}^{+0.2}$ \\
\hline 5112072 & $173.1_{-2.9}^{+2.6}$ & $35.1_{-0.6}^{+0.4}$ & $126.3_{-0.2}^{+0.2}$ \\
\hline 5112361 & $211.9_{-3.0}^{+3.5}$ & $21.4_{-0.5}^{+0.5}$ & $69.7_{-0.2}^{+0.2}$ \\
\hline 5112373 & $347.0_{-6.4}^{+5.8}$ & $14.6_{-0.4}^{+0.4}$ & $44.1_{-0.2}^{+0.2}$ \\
\hline 5112387 & $333.4_{-6.9}^{+6.0}$ & $14.3_{-0.5}^{+0.4}$ & $45.1_{-0.2}^{+0.2}$ \\
\hline 5112401 & $409.3_{-7.8}^{+8.8}$ & $14.0_{-0.4}^{+0.5}$ & $36.0_{-0.3}^{+0.2}$ \\
\hline 5112467 & $308.8_{-5.1}^{+5.4}$ & $14.4_{-0.4}^{+0.5}$ & $46.1_{-0.2}^{+0.2}$ \\
\hline 5112491 & $310.6_{-5.3}^{+4.9}$ & $14.3_{-0.4}^{+0.4}$ & $44.4_{-0.2}^{+0.2}$ \\
\hline 5112730 & $342.4_{-6.7}^{+7.4}$ & $13.9_{-0.6}^{+0.6}$ & $43.6_{-0.2}^{+0.2}$ \\
\hline 5112938 & $348.0_{-5.3}^{+5.8}$ & $13.9_{-0.4}^{+0.3}$ & $45.0_{-0.2}^{+0.2}$ \\
\hline 5112950 & $352.3_{-7.5}^{+6.9}$ & $13.1_{-0.6}^{+0.5}$ & $41.3_{-0.2}^{+0.3}$ \\
\hline 5112974 & $329.1_{-6.4}^{+6.4}$ & $13.0_{-0.5}^{+0.4}$ & $40.1_{-0.2}^{+0.2}$ \\
\hline 5113441 & $170.0_{-0.3}^{+0.4}$ & $47.0_{-0.4}^{+0.4}$ & $155.6_{-0.1}^{+0.1}$ \\
\hline 5200152 & $355.4_{-8.4}^{+7.0}$ & $14.8_{-0.5}^{+0.5}$ & $45.1_{-0.3}^{+0.3}$ \\
\hline
\end{tabular}

Table B.3. Same as in Table B.1 but for NGC 6811.

\begin{tabular}{cccr}
\hline \hline KIC ID & $\begin{array}{c}a_{\text {meso }} \\
(\mathrm{ppm})\end{array}$ & $\begin{array}{c}b_{\text {meso }} \\
(\mu \mathrm{Hz})\end{array}$ & \multicolumn{1}{c}{$\begin{array}{c}v_{\max } \\
(\mu \mathrm{Hz})\end{array}$} \\
\hline 9532903 & $173.8_{-2.1}^{+2.0}$ & $29.2_{-0.7}^{+0.7}$ & $93.9_{-0.4}^{+0.6}$ \\
9534041 & $150.5_{-0.9}^{+0.9}$ & $32.4_{-0.4}^{+0.5}$ & $111.8_{-0.3}^{+0.3}$ \\
9655101 & $163.6_{-1.2}^{+1.2}$ & $35.2_{-0.7}^{+0.7}$ & $101.3_{-0.4}^{+0.4}$ \\
9716090 & $189.5_{-0.8}^{+0.8}$ & $30.7_{-0.9}^{+0.7}$ & $109.6_{-0.2}^{+0.2}$ \\
9716522 & $244.2_{-4.3}^{+3.6}$ & $17.3_{-0.5}^{+0.4}$ & $54.1_{-0.3}^{+0.3}$ \\
9776739 & $178.0_{-2.3}^{+2.6}$ & $30.1_{-0.7}^{+0.7}$ & $94.8_{-0.6}^{+0.6}$ \\
\hline
\end{tabular}




\section{Appendix C: Predictions from scaling relations}

The resulting predictions of the scaling relations using the estimated parameters listed in Table 2 are shown in Fig. C. 1 for the models $\mathcal{M}_{a, 1}$ and $\mathcal{M}_{a, 2}$, and in Fig. C.2 for the models $\mathcal{M}_{b, 1}$ $\mathcal{M}_{b, 2}, \mathcal{M}_{b, 3}$, and $\mathcal{M}_{b, 4}$.

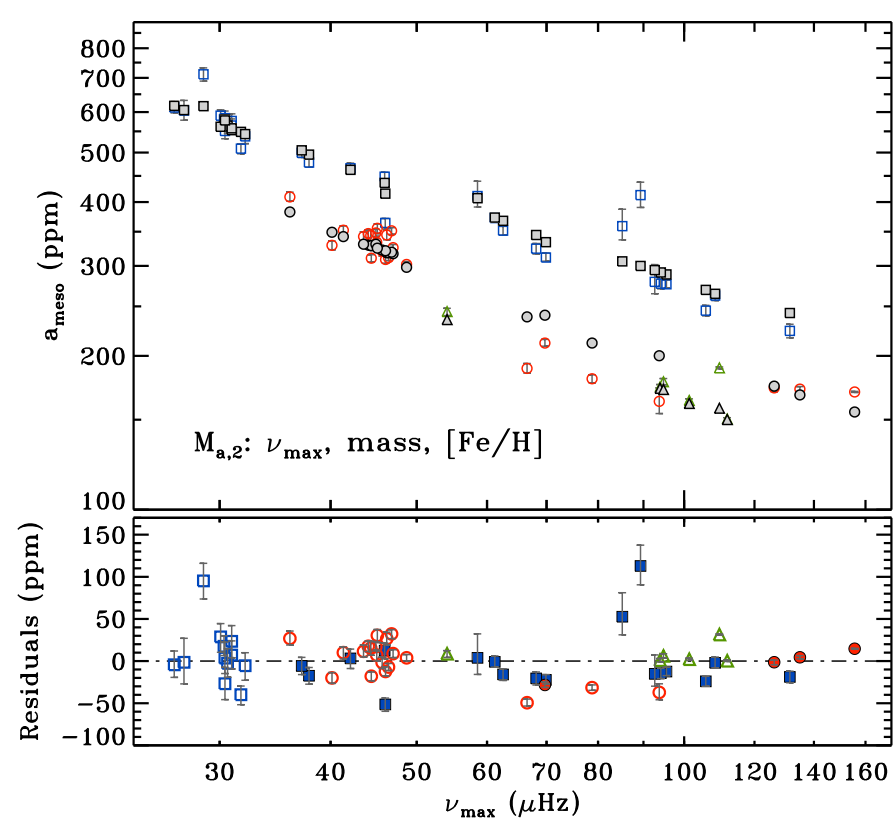

Fig. C.1. Top panels: predicted meso-granulation amplitudes (solid gray symbols) as a function of $v_{\max }$ for NGC 6791 (squares), NGC 6819 (circles) and NGC 6811 (triangles). Median values of the free parameters have been adopted for each scaling relation, as reported in Table 2. Observed meso-granulation amplitudes are shown in color with open symbols. The left plot shows the results for the model $\mathcal{M}_{a, 1}$, while the right one uses model $\mathcal{M}_{a, 2}$. Bottom panels: the residuals computed as (Observed-Predicted) meso-granulation amplitudes, with same color and symbol type as in Fig. 5a. Bayesian credible intervals of $68.3 \%$ are overlaid in both panels. 
A\&A 605, A3 (2017)
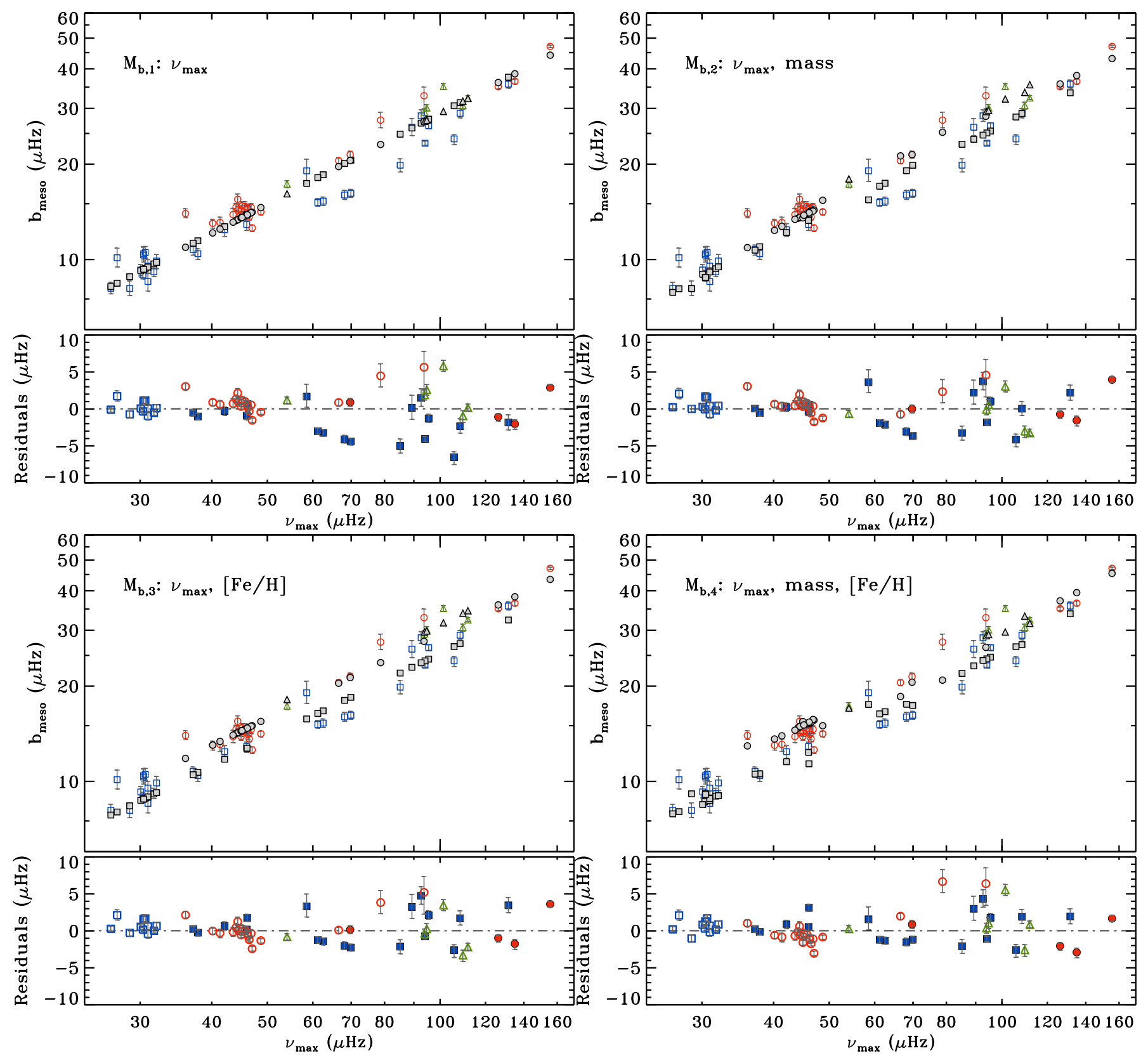

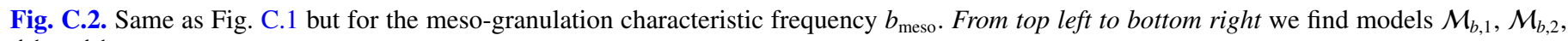
$\mathcal{M}_{b, 3}, \mathcal{M}_{b, 4}$. 